\title{
The low-energy limiting behavior of the pseudofermion dynamical theory
}

\author{
J.M.P. Carmelo a,b L.M. Martelo ${ }^{\text {b,c }}$, K. Penc ${ }^{\mathrm{d}, *}$ \\ a Department of Physics, Massachusetts Institute of Technology, Cambridge, MA 02139-4307, USA \\ ${ }^{\mathrm{b}}$ GCEP, Center of Physics, University of Minho, Campus Gualtar, P-4710-057 Braga, Portugal \\ c Physics Department, Engineering Faculty of University of Porto, P-4200-465 Porto, Portugal \\ ${ }^{\mathrm{d}}$ Research Institute for Solid State Physics and Optics, PO Box 49, H-1525 Budapest, Hungary
}

Received 1 September 2005; accepted 19 December 2005

Available online 18 January 2006

\begin{abstract}
In this paper we show that the general finite-energy spectral-function expressions provided by the pseudofermion dynamical theory for the one-dimensional Hubbard model lead to the expected low-energy Tomonaga-Luttinger liquid correlation function expressions. Moreover, we use the former general expressions to derive correlation-function asymptotic expansions in space and time which go beyond those obtained by conformal-field theory and bosonization: we derive explicit expressions for the pre-factors of all terms of such expansions and find that they have an universal form, as the corresponding critical exponents. Our results refer to all finite values of the on-site repulsion $U$ and to a chain of length $L$ very large and with periodic boundary conditions for the above model, but are of general nature for many integrable interacting models. The studies of this paper clarify the relation of the low-energy Tomonaga-Luttinger liquid behavior to the scattering mechanisms which control the spectral properties at all energy scales and provide a broader understanding of the unusual properties of quasi-one-dimensional nanostructures, organic conductors, and optical lattices of ultracold fermionic atoms. Furthermore, our results reveal the microscopic mechanisms which are behind the similarities and differences of the low-energy and finite-energy spectral properties of the model metallic phase.
\end{abstract}

(C) 2006 Elsevier B.V. All rights reserved.

PACS: 70.

\footnotetext{
* Corresponding author.

E-mail address: penc@szfki.hu (K. Penc).
} 


\section{Introduction}

Over the past twenty five years it has been shown that the low-energy physics of a variety of models of one-dimensional (1D) correlated electrons can be described by the TomonagaLuttinger liquid (TLL) theory [1]. Indeed, the low-energy physics of such interacting quantum problems displays universal properties which are also found in the simple and exactly solvable Tomonaga [2] and Luttinger [3] models. Importantly, the low-energy TLL universal behavior was observed in different real materials and systems, as for instance in carbon nanotubes [4,5], ballistic wires [6], quasi-1D organic conductors [7], 1D metallic chains [8], and quasi-1D quantum gases of ultracold fermionic atoms [9]. On the other hand, the low-energy phases of some quasi-1D compounds are not metallic and correspond to broken-symmetry states [10]. Recently, the resolution of photoemission experiments has improved, and the normal state of these compounds was found to display exotic spectral properties [11]. However, such a metallic phase refers to finite energies and is not described by the TLL theory.

The 1D Hubbard model is one of the few realistic models for correlated electrons in a discrete lattice for which one can exactly calculate all the energy eigenstates and their energies [12, 13]. It includes a first-neighbor transfer-integral $t_{1}$, for electron hopping along the chain, and an effective on-site Coulomb repulsion $U$. For finite-energy values, the metallic phase of this model is not a TLL and thus the study of spectral functions is a very involved many-electron problem. Fortunately, the recently introduced pseudofermion dynamical theory (PDT) provides explicit expressions for these functions [14,15]. Moreover, the theory describes successfully the unusual spectral features of quasi-1D compounds for the whole finite-energy band width [16]. More recently, consistent results were obtained by numerical techniques, involving the use of the dynamical density matrix renormalization group method [17]. Furthermore, when combined with the renormalization group, the use of the PDT reveals that a system of weakly coupled Hubbard chains is suitable for the successful description of the phase diagram observed in quasi-1D doped Mott-Hubbard insulators [18]. The PDT is a generalization for all values of $U / t_{1}$ of the method introduced in Ref. [19] for $U / t_{1} \rightarrow \infty$. Such an extension was fulfilled by means of the relation of the original electrons to the exotic objects whose occupancy configurations describe all energy eigenstates of the model [20]. The electron-rotated-electron unitary transformation [20], defined in the whole Hilbert space, and the pseudoparticle-pseudofermion unitary transformation [14, $15,21]$, defined in the subspace where the one- and two-electron excitations are contained, play a major role in the construction of the PDT.

In turn, the low-energy physics of the model corresponds to the universal TLL behavior and was studied by different techniques, such as bosonization [22] and conformal-field theory [23, 24]. There are many investigations where the low-energy conformal invariance was combined with the model exact Bethe-ansatz solution in the study of the asymptotics of correlation functions and related quantities [25-34].

The connection of the low-energy TLL behavior to the microscopic scattering mechanisms which control the unusual spectral properties of the model at all energy scales $[14,21]$ remains an interesting open problem, which we study in this paper. Indeed, while conformal-field theory and bosonization techniques do not provide correlation-function expressions for finite energy, we show here that the general finite-energy PDT introduced in Refs. $[14,15]$ reproduces the expected correlation-function expressions in the limit of low energy. Moreover, we derive the corresponding correlation-function asymptotic expansions in space and time. Such expansions go beyond those obtained by conformal-field theory and bosonization: we derive explicit expressions for the pre-factors of all terms of such expansions and find that they have an universal form, as 
the corresponding critical exponents. We also find the relation of the low-energy pseudofermion description to the conformal-field theory primary fields and Virasoro-algebra generators [23,24].

In this paper the emergence of the TLL low-energy physics is described in terms of the general non-perturbative microscopic scattering mechanisms of the model at all energy scales $[14,15,21]$. Thus, our results provide further information about the microscopic mechanisms and scattering processes behind both the low-energy and finite-energy properties of one-dimensional fermionic interacting problems. For instance, we clarify why there occurs a different type of momentum and energy dependence for the low-energy and finite-energy parts of important singular spectral features of the model metallic phase. Furthermore, our findings lead to a broader understanding of the unusual properties observed in low-dimensional materials and nanostructures [4-8] and systems of interacting ultracold fermionic atoms in 1D optical lattices [35]. Following the investigations on quasi-1D quantum gases of ultracold fermionic atoms [9], studies about twoatom correlation functions of interacting ultracold fermionic atoms in 1D optical lattices are in progress [36]. Recently, the model was used in preliminary theoretical investigations of the density profiles and collective models of 1D ultracold fermionic atoms confined in an optical lattice with harmonic trapping potential [37].

Our study provides the details of the preliminary results on the universal form of the prefactors of the correlation-function asymptotic expansions presented in short form in Ref. [38]. The paper is organized as follows: in Section 2 we introduce the model and summarize the basic information about the PDT which is needed for our studies. The general finite-energy PDT spectral-function expressions are shown in Section 3 to recover in the limit of low energy the correct correlation-function expressions and corresponding asymptotic expansions in space and time. Moreover, we are able to obtain expressions for the pre-factors of all terms of such expansions. In Section 4 we discuss the universal form of the pre-factors of all terms of the correlation-function asymptotic expansions and the emergence of the TLL low-energy physics in terms of the general scattering mechanisms which control the model spectral properties at all energy scales. Furthermore, in that section we discuss the qualitative difference between the low-energy and finite-energy parts of the singular charge and spin spectral features of the metallic phase and the relation of the low-energy pseudofermion description to the conformal-field theory primary fields and Virasoro-algebra generators. Finally, the concluding remarks are presented in Section 5 .

\section{The model, the general correlation functions, and the pseudofermions}

The 1D Hubbard model reads

$$
\hat{H}=-t_{1} \sum_{j, \sigma}\left[c_{j, \sigma}^{\dagger} c_{j+1, \sigma}+\text { h.c. }\right]+U \sum_{j} \hat{n}_{j, \uparrow} \hat{n}_{j, \downarrow},
$$

where $c_{j, \sigma}^{\dagger}$ and $c_{j, \sigma}$ are spin-projection $\sigma=\uparrow, \downarrow$ electron operators at site $j=1,2, \ldots, N_{a}$ and $\hat{n}_{j, \sigma}=c_{j, \sigma}^{\dagger} c_{j, \sigma}$. The model (1) describes $N_{\uparrow}$ spin-up electrons and $N_{\downarrow}$ spin-down electrons in a chain of $N_{a}$ sites. We denote the electronic number by $N=N_{\uparrow}+N_{\downarrow}$. The number of lattice sites $N_{a}$ is even and very large. For simplicity, we use units such that both the lattice spacing $a$ and the Planck constant are one. In these units the chain length reads $L=N_{a}$. Our results refer to periodic boundary conditions. We consider an electronic density $n=n_{\uparrow}+n_{\downarrow}$ in the range $0<n<1$ and a spin density $m=n_{\uparrow}-n_{\downarrow}$ such that $0<m<n$, where $n_{\sigma}=N_{\sigma} / L$ and $\sigma=\uparrow, \downarrow$. We introduce the Fermi momenta which except for $1 / L$ corrections are given by $\pm k_{F \sigma}= \pm \pi n_{\sigma}$ 
and $\pm k_{F}= \pm\left[k_{F \uparrow}+k_{F \downarrow}\right] / 2= \pm \pi n / 2$. The Hamiltonian (1) commutes with the generators of the $\eta$-spin and spin $S U(2)$ algebras $[20,39,40]$. We call the $\eta$-spin and spin eigenvalues of the energy eigenstates $\eta$ and $S$, respectively, and the corresponding projections $\eta_{z}$ and $S_{z}$.

We consider the following general $\mathcal{N}$-electron correlation function

$$
\chi_{\mathcal{N}^{(}}^{l}(k, \omega)=l \int_{-\infty}^{+\infty} d \omega^{\prime} \frac{B_{\mathcal{N}^{\prime}}^{l}\left(k, \omega^{\prime}\right)}{\omega-\omega^{\prime}+i l 0}
$$

where $B_{\mathcal{N}}^{l}(k, \omega)$ is the corresponding $\mathcal{N}$-electron spectral function given by

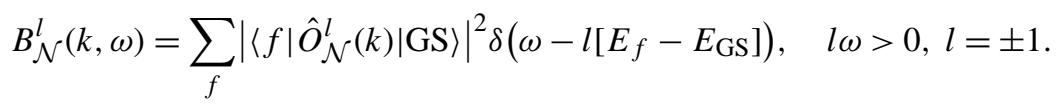

Here the general $\mathcal{N}$-electron operators $\hat{O}_{\mathcal{N}}^{+1}(k) \equiv \hat{O}_{\mathcal{N}}^{\dagger}(k)$ and $\hat{O}_{\mathcal{N}}^{-1}(k) \equiv \hat{O}_{\mathcal{N}}(k)$ carry momentum $k$, the $f$ summation runs over the excited energy eigenstates, the energy $E_{f}$ corresponds to these states, and $E_{\mathrm{GS}}$ is the ground-state energy. Most common examples are the operator $\hat{O}_{1}(k)=c_{k, \sigma}$ and different choices of charge, spin, and Cooper-pair $\mathcal{N}=2$ operators. For simplicity, we use in expression (3) a momentum extended scheme such that $k \in(-\infty,+\infty)$, yet it is a simple exercise to obtain the corresponding spectral function expressions for the first Brillouin zone.

The double Fourier transform $\tilde{\chi}_{\mathcal{N}}^{l}(x, t)$ of the general correlation function (2) relative to the momentum $k$ and energy $\omega$ can be expressed in terms of the corresponding Fourier transform $\tilde{B}_{\mathcal{N}^{l}}^{l}(x, t)$ of the spectral function (3) as

$$
\tilde{\chi}_{\mathcal{N}}^{l}(x, t)=-i 2 \pi \theta(l t) \tilde{B}_{\mathcal{N}}^{l}(x, t),
$$

where here and in other expressions provided below $\theta(y)=1$ for $y>0$ and $\theta(y)=0$ for $y \leqslant 0$.

One of the goals of this paper is the evaluation of a general asymptotic expansion for the correlation function (4). To reach such a goal, in Section 3 we use the finite-energy expressions derived in Ref. [14] for the general spectral function (3) by means of the PDT. The pseudofermion description is related to the holon and spinon representation for the model: all its energy eigenstates can be described in terms of occupancy configurations of $\eta$-spin $1 / 2$ holons, spin $1 / 2$, spinons, and $\eta$-spin-less and spin-less $c$ pseudoparticles [20]. We use the notation $\pm 1 / 2$ holons and $\pm 1 / 2$ spinons according to the values of the $\eta$-spin and spin projections, respectively. For large values of $U / t_{1}$, the $+1 / 2$ holons and $-1 / 2$ holons become the holons and doublons, respectively, used in the studies of Ref. [41]. The electron-rotated-electron unitary transformation [20] maps the electrons onto rotated electrons such that rotated-electron double occupation, unoccupation, and spin-up and spin-down single occupation are good quantum numbers for all values of $U$. The $\pm 1 / 2$ holons of charge $\pm 2 e$ and zero spin and the charge-less $\pm 1 / 2$ spinons are generated from the electrons by that unitary transformation, where $-e$ denotes the electronic charge. The corresponding holon and spinon number operators $\hat{M}_{c, \pm 1 / 2}$ and $\hat{M}_{s, \pm 1 / 2}$, respectively, are of the form given in Eq. (24) of Ref. [20] and involve the electron-rotated-electron unitary operator.

While the $-1 / 2$ and $+1 / 2$ holons refer to the rotated-electron doubly occupied and unoccupied sites, respectively, the $-1 / 2$ and $+1 / 2$ spinons correspond to the spin degrees of freedom of the spin-down and spin-up rotated-electron singly occupied sites, respectively. The charge degrees of freedom of the latter sites are described by the spin-less and $\eta$-spin-less $c$ pseudoparticles, which are composite objects of a chargeon and a antichargeon, and thus carry charge $-e$ or $+e$ for the description of the transport of charge in terms of electrons and electronic holes, 
respectively [20]. The $c v$ pseudoparticles (and $s v$ pseudoparticles) such that $v=1,2, \ldots$ are $\eta$ spin singlet (and spin singlet) $2 v$-holon (and $2 v$-spinon) composite objects. Thus, the numbers of $\pm 1 / 2$ holons $(\alpha=c)$ and $\pm 1 / 2$ spinons $(\alpha=s)$ read

$$
M_{\alpha, \pm 1 / 2}=L_{\alpha, \pm 1 / 2}+\sum_{\nu=1}^{\infty} v N_{\alpha \nu}, \quad \alpha=c, s,
$$

where $N_{\alpha \nu}$ denotes the number of $\alpha v$ pseudoparticles and $L_{c, \pm 1 / 2}=\left[\eta \mp \eta_{z}\right]$ and $L_{s, \pm 1 / 2}=$ $\left[S \mp S_{z}\right.$ ] gives the number of $\pm 1 / 2$ Yang holons and $\pm 1 / 2$ HL spinons, respectively. Those are the holons and spinons that are not part of composite pseudoparticles. The total number of holons $(\alpha=c)$ and spinons $(\alpha=s)$ is given by

$$
M_{\alpha}=\left[M_{\alpha,+1 / 2}+M_{\alpha,-1 / 2}\right], \quad \alpha=c, s .
$$

All energy eigenstates can be described by occupancy configurations of $c$ pseudoparticles, $\alpha \nu$ pseudoparticles, $-1 / 2$ Yang holons, and $-1 / 2$ HL spinons [20]. For the ground state, $N_{c}=N$, $N_{s 1}=N_{\downarrow}$, and $N_{c v}=N_{s v^{\prime}}=L_{\alpha,-1 / 2}=0$ for $\alpha=c, s, v>0$, and $v^{\prime}>0$.

The construction of the PDT involves a second unitary transformation, which maps the $c$ pseudoparticles (and composite $\alpha \nu$ pseudoparticles) onto $c$ pseudofermions (and composite $\alpha v$ pseudofermions) [14,15,21]. Such a transformation introduces shifts of order $1 / L$ in the pseudoparticle discrete momentum values and leaves all other pseudoparticle properties invariant. As a result of such momentum shifts and in contrast to the $c$ pseudoparticles and composite $\alpha v$ pseudoparticles, the corresponding pseudofermions have no residual-interaction energy terms.

A concept widely used in the PDT is that of a CPHS ensemble subspace [14,42]. (Here CPHS stands for $c$ pseudofermion, holon, and spinon.) Such a subspace is spanned by all energy eigenstates with fixed values for the $-1 / 2$ Yang holon number $L_{c,-1 / 2},-1 / 2 \mathrm{HL}$ spinon number $L_{s,-1 / 2}, c$ pseudofermion number $N_{c}$, and for the sets of $\alpha v$ pseudofermion numbers $\left\{N_{\alpha \nu}\right\}$ corresponding to the composite pseudofermion branches.

\section{General asymptotic expressions of correlation functions}

Here we derive the pre-factors of all terms of the general asymptotic expansion for the correlation function (4) by use the finite-energy spectral-function expressions derived in Refs. [14,15] by means of the PDT. To reach such a goal, we start by defining the low-energy subspace for the electronic densities and spin densities considered in this paper [43] and providing further information about the pseudofermion description when defined in such a subspace.

\subsection{Pseudofermion description in the low-energy subspace}

For each correlation function, the electronic number deviations $\Delta N_{\uparrow}$ and $\Delta N_{\downarrow}$ have welldefined values and for electronic densities $0<n<1$ and spin densities $0<m<n$, all low-energy excited energy eigenstates belong to a single CPHS ensemble subspace such that

$$
\begin{aligned}
& \Delta N_{c}=\Delta N, \quad \Delta N_{s 1}=\Delta N_{\downarrow}, \\
& \left\{N_{c v}\right\}=\left\{N_{s v^{\prime}}\right\}=\left\{L_{c,-1 / 2}\right\}=\left\{L_{s,-1 / 2}\right\}=0, \quad v=1,2,3, \ldots, v^{\prime}=2,3, \ldots
\end{aligned}
$$

The results of this section refer to such a correlation-function low-energy subspace. 
For simplicity, in this paper we denote the $s 1$ branch by $s$ branch. The $c$ and $s$ indices used here correspond to the $c 0$ and $s 1$ indices in all quantities of Refs. [14,15,21,42]. In our study we use the index $\iota= \pm 1$, which refers to the right $(\iota=+1)$ and left $(\iota=-1) \alpha, \iota$ Fermi points. For the ground state and except for $1 / L$ corrections [20] such points read $\iota q_{F c}^{0}=\iota 2 k_{F}$ and $\iota q_{F s}^{0}=\iota k_{F \downarrow}$. All the $\alpha$ (and $\iota$ ) sums and products appearing in the expressions provided throughout this paper run over the values $\alpha=c, s$ (and $\iota=+1,-1$ ). The $\alpha$ pseudofermion number deviation $\Delta N_{\alpha}$ and current deviation $\Delta J_{\alpha}^{F}$ of the excited energy eigenstates relative to the initial ground state are given by

$$
\Delta N_{\alpha}=\sum_{\iota} \Delta N_{\alpha, \iota}^{F}, \quad \Delta J_{\alpha}^{F}=\frac{1}{2} \sum_{\iota} \iota \Delta N_{\alpha, \iota}^{F}, \quad \alpha=c, s,
$$

where

$$
\Delta N_{\alpha, \iota}^{F}=\Delta N_{\alpha, \iota}^{0, F}+\iota Q_{\alpha}^{0} / 2 \pi, \quad \alpha=c, s, \iota= \pm 1 .
$$

Here $\Delta N_{\alpha, l}^{0, F}$ stands for the number of $\alpha$ pseudofermions created $\left(\Delta N_{\alpha, l}^{0, F}>0\right)$ or annihilated $\left(\Delta N_{\alpha, l}^{0, F}<0\right)$ as a result of the ground-state-excited-energy-eigenstate transition and $Q_{\alpha}^{0} / 2$ is a scattering-less phase shift that has a single and well-defined value for the correlation-function excitation CPHS subspace such that

$$
\begin{aligned}
& Q_{c}^{0} / 2=0, \quad \Delta N_{s} \text { even, } \quad Q_{c}^{0} / 2= \pm \pi / 2, \quad \Delta N_{s} \text { odd }, \\
& Q_{s}^{0} / 2=0, \quad \Delta N_{c}+\Delta N_{s} \text { even, } \quad Q_{s}^{0} / 2= \pm \pi / 2, \quad \Delta N_{c}+\Delta N_{s} \text { odd. }
\end{aligned}
$$

It is useful for our study to consider the pseudofermion subspace (PS). It is spanned by an initial ground state $|\mathrm{GS}\rangle$ and all excited energy eigenstates contained in the one- two-electron excitations $[14,15]$. The pseudoparticle-pseudofermion unitary transformation which maps the $\alpha$ pseudoparticle onto the $\alpha$ pseudofermion is defined in the PS. The $\alpha$ pseudoparticle has discrete bare-momentum values $q_{j}=[2 \pi / L] I_{j}^{\alpha}$ such that $I_{j}^{\alpha}$ are consecutive integers or half-odd integers [20]. These values are good quantum numbers whose allowed occupancies are one and zero only [20]. Due to the values of such quantum numbers, the current deviations $\Delta J_{\alpha}^{F}$ are integers or halfodd integers depending on the parities of the number deviations $\Delta N=\Delta N_{c}$ and $\Delta N_{\downarrow}=\Delta N_{s}$ as follows

$$
\begin{aligned}
& \Delta J_{c}^{F}=\frac{\Delta N_{c}+\Delta N_{s}}{2} \bmod 1=\frac{\Delta N+\Delta N_{\downarrow}}{2} \bmod 1, \\
& \Delta J_{s}^{F}=\frac{\Delta N_{c}}{2} \bmod 1=\frac{\Delta N}{2} \bmod 1 .
\end{aligned}
$$

On the other hand, the $\alpha$ pseudofermion has discrete canonical-momentum values given by $[14,21]$,

$$
\bar{q}_{j}=\bar{q}\left(q_{j}\right)=q_{j}+Q_{\alpha}^{\Phi}\left(q_{j}\right) / L, \quad \alpha=c, s,
$$

where $j=1,2, \ldots, N_{\alpha}^{*}$ and the number $N_{\alpha}^{*}$ is such that $N_{\alpha}^{*}=N_{\alpha}+N_{\alpha}^{h}$. Here $N_{\alpha}^{h}$ denotes the number of $\alpha$ pseudofermion (and $\alpha$ pseudoparticle) holes [14,15,20]. For the PS low-energy sector such numbers are given by

$$
\begin{aligned}
& N_{c}^{*}=N_{a}, \quad N_{s}^{*}=N_{\uparrow}^{0}+\Delta N_{\uparrow}, \\
& N_{c}^{h}=N_{a}-N^{0}-\Delta N, \quad N_{s}^{h}=N_{\uparrow}^{0}+\Delta N_{\uparrow}-N_{\downarrow}^{0}-\Delta N_{\downarrow} .
\end{aligned}
$$


Thus, the bare-momentum values are defined in the range $-q_{\alpha}^{0} \leqslant q \leqslant+q_{\alpha}^{0}$ where the limiting bare-momentum $q_{\alpha}^{0}$ reads

$$
q_{c}^{0}=\pi, \quad q_{s}^{0}=k_{F \uparrow} .
$$

In these expressions we have neglected $1 / L$ corrections [20]. When below we refer to the $\alpha$ pseudofermion bare-momentum $q$, we mean that $q$ is the bare-momentum value that corresponds to the pseudofermion canonical momentum $\bar{q}=q+Q_{\alpha}^{\Phi}(q) / L$. Except for the discrete momentum values, the above pseudoparticle and pseudofermion have the same properties. Thus, all the energy eigenstates that span the low-energy sector of the PS can described by occupancy configurations of $\alpha$ pseudofermions. The functional

$$
Q_{\alpha}^{\Phi}\left(q_{j}\right) / 2=\pi \sum_{\alpha^{\prime}} \sum_{j=1}^{N_{\alpha}^{*}} \Phi_{\alpha \alpha^{\prime}}\left(q_{j}, q_{j^{\prime}}\right) \Delta N_{\alpha^{\prime}}\left(q_{j^{\prime}}\right), \quad \alpha=c, s,
$$

of Eq. (12) is the scattering part of the overall pseudofermion or pseudofermion hole phase shift [21]

$$
Q_{\alpha}(q) / 2=Q_{\alpha}^{0} / 2+Q_{\alpha}^{\Phi}(q) / 2, \quad \alpha=c, s,
$$

where $Q_{\alpha}^{0} / 2$ is the scattering-less phase shift given in Eq. (10). Such an overall phase shift plays an important role in the $\alpha$ pseudofermion scattering theory and related spectral properties. On the right-hand side of Eq. (15), $\Delta N_{\alpha}\left(q_{j}\right) \equiv N_{\alpha}\left(q_{j}\right)-N_{\alpha}^{0}\left(q_{j}\right)$ is the excited-state $\alpha$ branch bare-momentum distribution-function deviation relative to the initial ground state value and $\pi \Phi_{\alpha \alpha^{\prime}}\left(q, q^{\prime}\right)$ is a two-pseudofermion phase shift such that the $\alpha^{\prime}$ (and $\alpha$ ) pseudofermion or hole of momentum $q^{\prime} \in\left[-q_{\alpha^{\prime}}^{0},+q_{\alpha^{\prime}}^{0}\right]$ (and $q \in\left[-q_{\alpha}^{0},+q_{\alpha}^{0}\right]$ ) is the scattering center created under the ground-state-excited-state transition (and the scatterer) [21]. The two-pseudofermion phase shifts $\pi \Phi_{\alpha \alpha^{\prime}}\left(q, q^{\prime}\right)$ are defined in Appendix A.

The low-energy correlation-function CPHS subspace contains several J-CPHS subspaces. The current deviation values of the energy eigenstates which span each of the latter subspaces differ in at least one of the two current deviation values $\left\{\Delta J_{c}^{F}, \Delta J_{s}^{F}\right\}$. At low-energy, the reduced JCPHS subspaces considered in Ref. [14] are spanned by a single energy eigenstate. Since such a state is the lowest-energy eigenstate of the corresponding J-CPHS subspace, we call it J-ground state. It corresponds to a $c$ and $s$ pseudofermion bare-momentum densely packed occupancy configuration such that $q_{F \alpha,-1} \leqslant q \leqslant q_{F \alpha,+1}$. Here the J-ground-state Fermi point $q_{F \alpha, \iota}$ reads

$$
\begin{aligned}
& q_{F \alpha, \iota}=\iota q_{F \alpha}^{0}+\Delta q_{F \alpha, \iota}=q_{F \alpha, \iota}^{0}+\frac{Q_{\alpha}^{0}}{L}, \quad q_{F c}^{0}=2 k_{F}, \quad q_{F s}^{0}=k_{F \downarrow}, \\
& \alpha=c, s, \iota= \pm 1,
\end{aligned}
$$

where we have neglected $1 / L$ corrections to the value of $q_{F \alpha}^{0}[20]$ and $\Delta q_{F \alpha, \iota}$ denotes the $\alpha, \iota$ bare-momentum Fermi-point deviation relative to the corresponding ground-state value given by

$$
\Delta q_{F \alpha, \iota}=\Delta q_{F \alpha, \iota}^{0}+\frac{Q_{\alpha}^{0}}{L}=\iota \frac{2 \pi}{L} \Delta N_{\alpha, \iota}^{F}=\iota \frac{2 \pi}{L}\left[\frac{\Delta N_{\alpha}}{2}+\iota \Delta J_{\alpha}^{F}\right], \quad \alpha=c, s, \iota= \pm 1 .
$$

In these expressions the bare-momentum $q_{F \alpha, \iota}^{0}$ and corresponding deviation $\Delta q_{F \alpha, \iota}^{0}$ read

$$
q_{F \alpha, \iota}^{0}=\iota q_{F \alpha}^{0}+\Delta q_{F \alpha, \iota}^{0}, \quad \Delta q_{F \alpha, \iota}^{0}=\iota \frac{2 \pi}{L} \Delta N_{\alpha, \iota}^{0, F}, \quad \alpha=c, s, \iota= \pm 1 .
$$


A J-ground state has excitation momentum

$$
k_{0}^{F}=\sum_{\alpha} \Delta P_{\alpha}^{F},
$$

where

$$
\Delta P_{\alpha}^{F}=\sum_{\iota} \iota q_{F \alpha}^{0} \Delta N_{\alpha, \iota}^{F}=2 q_{F \alpha}^{0} \Delta J_{\alpha}^{F}, \quad \alpha=c, s .
$$

Such a state is generated from the ground state by zero-energy and finite-momentum elementary processes (B), which create or annihilate $\alpha$ pseudofermions at or from the four $\alpha, \iota$ Fermi points, respectively. In turn, the PDT processes (A) do not exist at low energy [14]. The corresponding $\mathrm{J}$-CPHS subspace is spanned by energy eigenstates generated from the J-ground state by smallmomentum and low-energy processes in the vicinity of the $\alpha, \iota$ Fermi points, which we call elementary processes (C). Such processes conserve the set of $\left\{\Delta N_{c}, \Delta N_{s}, \Delta J_{c}^{F}, \Delta J_{s}^{F}\right\}$ deviation values. For each low-energy J-CPHS subspace, the general momentum spectrum provided in Eq. (29) of Ref. [14] simplifies and is single valued and given in Eq. (20).

A crucial point for the low-energy scattering properties and corresponding correlationfunction asymptotic expansions studied in this paper is that the $\alpha$ pseudofermions and holes created by the above processes $(\mathrm{C})$ are not active scattering centers, once the phase shifts generated by the created pseudofermions exactly cancel those originated by creation of the corresponding holes [14]. It follows that the overall scattering phase shift (15) has for each $\alpha$ pseudofermion or hole scatterer of momentum $q$ the same value

$$
\begin{aligned}
Q_{\alpha}^{\Phi}(q) / 2 & =\pi \sum_{\alpha^{\prime}} \sum_{\iota^{\prime}} \Phi_{\alpha \alpha^{\prime}}\left(q, \iota^{\prime} q_{F \alpha^{\prime}}^{0}\right) \Delta N_{\alpha^{\prime}, \iota^{\prime}}^{F} \\
& =\pi \sum_{\alpha^{\prime}} \sum_{\iota^{\prime}} \Phi_{\alpha \alpha^{\prime}}\left(q, \iota^{\prime} q_{F \alpha^{\prime}}^{0}\right)\left[\frac{\Delta N_{\alpha^{\prime}}}{2}+\iota^{\prime} \Delta J_{\alpha^{\prime}}^{F}\right], \quad \alpha=c, s,
\end{aligned}
$$

for all excited states spanning given J-CPHS subspace.

Note that the scattering part of the overall phase shift, Eq. (15), vanishes and is finite for the initial ground state and excited states, respectively. Thus, the ground-state-excited-energyeigenstate transition leads to a shift

$$
\Delta \bar{q}_{F \alpha, \iota}=\Delta q_{F \alpha, \iota}+Q_{\alpha}^{\Phi}\left(\iota q_{F \alpha}^{0}\right) / L=\Delta q_{F \alpha, \iota}^{0}+Q_{\alpha}\left(\iota q_{F \alpha}^{0}\right) / L, \quad \alpha=c, s, \iota= \pm 1,
$$

in the value of the four $\alpha, \iota$ canonical-momentum Fermi-points. Such a shift is the excited-state deviation in the value of $\bar{q}$ corresponding to $\bar{q}=q=\iota q_{F \alpha}^{0}$ for the initial ground state. The square of these shifts in units of $2 \pi / L$ plays a key-role in the spectral properties at all energy scales and is denoted by $2 \Delta_{\alpha}^{\iota}$. It can be written as follows

$$
2 \Delta_{\alpha}^{\iota} \equiv\left(\frac{\Delta \bar{q}_{F \alpha, \iota}}{[2 \pi / L]}\right)^{2}=\left(\iota \Delta N_{\alpha}^{0, F}+\frac{Q_{\alpha}\left(\iota q_{F \alpha}^{0}\right)}{2 \pi}\right)^{2}, \quad \alpha=c, s, \iota= \pm 1
$$

The general expression in terms of two-pseudofermion phase shifts of the functional $Q_{\alpha}\left(\iota q_{F \alpha}^{0}\right) / 2$ appearing in the second expression of Eq. (24) is given in Eqs. (35)-(37) of Ref. [14]. However, for the excited energy eigenstates that span the low-energy subspace the finite-energy deviation given in Eq. (14) of that reference vanishes. This property together with the values of the numbers given in Eq. (7) implies that at low energy the general expression of the general functional (24) 
simplifies to

$$
\begin{aligned}
2 \Delta_{\alpha}^{\iota} & =2 \Delta_{\alpha}^{\iota}\left(\Delta N_{c}, \Delta N_{s}, \Delta J_{c}^{F}, \Delta J_{s}^{F}\right)=\left(\sum_{\alpha^{\prime}}\left[\iota \xi_{\alpha \alpha^{\prime}}^{0} \frac{\Delta N_{\alpha^{\prime}}^{F}}{2}+\xi_{\alpha \alpha^{\prime}}^{1} \Delta J_{\alpha^{\prime}}^{F}\right]\right)^{2}, \\
\alpha & =c, s, \iota= \pm 1,
\end{aligned}
$$

where the parameters $\xi_{\alpha \alpha^{\prime}}^{j}$ can be expressed in terms of the two-pseudofermion phase shifts as follows

$$
\xi_{\alpha \alpha^{\prime}}^{j}=\delta_{\alpha, \alpha^{\prime}}+\sum_{\iota= \pm 1}\left(\iota^{j}\right) \Phi_{\alpha \alpha^{\prime}}\left(q_{F \alpha}^{0}, \iota q_{F \alpha^{\prime}}^{0}\right), \quad j=0,1, \alpha, \alpha^{\prime}=c, s .
$$

Expressions (25) and (26) are consistent with the low-energy form of the scattering phase shift given in Eq. (22).

The overall phase shift (22) controls the unusual spectral properties of the model through the pseudofermions anticommutators [14,21]. To illustrate the dependence of the latter anticommutators on the overall phase shifts for the two pseudofermion branches used in our study, let us consider pseudofermion creation and annihilation operators $f_{\bar{q}, \alpha}^{\dagger}$ and $f_{\bar{q}^{\prime}, \alpha}$, respectively. When the canonical momentum values $\bar{q}$ and $\bar{q}^{\prime}=q^{\prime}$ correspond to an excited-energy-eigenstate and the initial ground-state J-CPHS ensemble subspaces, respectively, the pseudofermion anticommutation relations read $[14,15]$,

$$
\left\{f_{\bar{q}, \alpha}^{\dagger}, f_{q^{\prime}, \alpha^{\prime}}\right\}=\delta_{\alpha, \alpha^{\prime}} \frac{1}{N_{\alpha}^{*}} e^{-i\left(\bar{q}-q^{\prime}\right) / 2} e^{i Q_{\alpha}(q) / 2} \frac{\sin \left(Q_{\alpha}(q) / 2\right)}{\sin \left(\left[\bar{q}-q^{\prime}\right] / 2\right)}, \quad \alpha, \alpha^{\prime}=c, s,
$$

and $\left\{f_{\bar{q}, \alpha}^{\dagger}, f_{q^{\prime}, \alpha^{\prime}}^{\dagger}\right\}=\left\{f_{\bar{q}, \alpha}, f_{q^{\prime}, \alpha^{\prime}}\right\}=0$. Here $N_{\alpha}^{*}$ is the number whose value is given in Eq. (13). The anticommutation relations (27) are indeed controlled by the value of the overall phase shift (16), which in our case has the same value for all excited energy eigenstates spanning a given J-CPHS subspace.

In addition to the overall phase shift (16), the group velocities

$$
v_{\alpha}(q)=\frac{\partial \epsilon_{\alpha}(q)}{\partial q}, \quad v_{\alpha} \equiv v_{\alpha}\left(q_{F \alpha}^{0}\right), \quad \alpha=c, s,
$$

play an important role in our studies. Here $\epsilon_{c}(q)$ and $\epsilon_{s}(q)$ are the $c$ and $s$ pseudofermions energy dispersions defined by Eqs. (C.15) and (C.16) of Ref. [20], respectively. These energy bands are plotted in Figs. 6 and 7 of Ref. [42], respectively, as a function $q$ for several values of $U / t_{1}$ and $n$ for $m=0$.

\subsection{The asymptotic expressions of correlation functions}

Our starting point for the study of low-energy correlation functions and associated correlationfunction asymptotic expansions in space $x$ and time $t$ is the general expression for the $\mathcal{N}$-electron spectral function (3) given in Eq. (41) of Ref. [14]. Fortunately, such a general expression simplifies for the low-energy problem considered here. Indeed, the numbers $N_{c 0}^{\mathrm{phNF}} \equiv N_{c}^{\mathrm{phNF}}$ and $N_{s 1}^{\mathrm{phNF}} \equiv N_{s}^{\mathrm{phNF}}$ of the summation on the right-hand side of the above equation vanish in our case because the corresponding bare-momentum distribution function deviation given in Eq. (14) of the same reference vanishes in the low-energy limit considered here. Also the numbers $N_{\alpha \nu, l}^{F}$ of the summation of the former equation vanish. This follows from the number values of Eq. (7), 
since the numbers $N_{\alpha \nu, \iota}^{F}$ refer to the $c v$ and $s v^{\prime}$ pseudofermion branches such that $v>0$ and $v^{\prime}>1$, respectively.

Furthermore, as discussed above, for each specific correlation function the low-energy subspace is contained in a single CPHS ensemble subspace, spanned by excited energy eigenstates with the same values for the number deviations $\Delta N_{c} \equiv \Delta N_{c 0}=\Delta N$ and $\Delta N_{s} \equiv \Delta N_{s 1}=\Delta N_{\downarrow}$. Thus, the $\left\{\Delta N_{\alpha \nu}\right\} \equiv\left\{\Delta N_{\alpha}\right\}$ summation of Eq. (41) of Ref. [14] is also absent, since the corresponding deviations have the same values for all excited states. This result together with the deviation expressions provided in Eq. (8) shows that the $\left\{\Delta N_{\alpha \nu, l}^{F}\right\} \equiv\left\{\Delta N_{\alpha, l}^{F}\right\}$ summation in expression (41) of Ref. [14] should be replaced by a corresponding $\left\{\Delta J_{\alpha}^{F}\right\} \equiv\left\{\Delta J_{c}^{F}, \Delta J_{s}^{F}\right\}$ summation over the low-energy J-CPHS ensemble subspaces contained in the correlation-function low-energy CPHS ensemble subspace.

It follows from all the above simplifications that for low-energy the expression (41) of Ref. [14] can be rewritten as

$$
B_{\mathcal{N}}^{l}(k, \omega)=\sum_{i=0}^{\infty} c_{i}^{l} \sum_{\left\{\Delta J_{\alpha}^{F}\right\}} B^{l, 0}(k, \omega), \quad c_{0}^{l}=1 .
$$

When expressed in terms of rotated-electron creation and annihilation operators, the $\mathcal{N}$ electron operator $\hat{O}_{\mathcal{N}}^{l}(k)$ of the general spectral-function expression (3) is given by a sum of operators, each corresponding to one of the integer $i$ values of the sum on the right-hand side of Eq. (29). The corresponding $i=0$ operator has the same expression in terms of rotated-electron creation and annihilation operators as $\hat{O}_{\mathcal{N}^{(}}^{l}(k)$ in terms of electronic creation and annihilation operators [14,15], respectively. In turn, the $i>0$ operators have a number of extra pairs of rotated-electron creation and annihilation operators which increases for increasing values of $i$.

For all values of $U / t_{1}$, the $i>0$ terms of expression (29) correspond to less than $1 \%$ of the total $\mathcal{N}$-electron spectral weight $[14,15,44]$. However, we have kept these terms and used the method presented below for the derivation of the general asymptotic expansion of the correlation function $\tilde{\chi}_{\mathcal{N}}^{l}(x, t)$ given in Eq. (4). The result is that the leading terms in the asymptotic expansion of such a function are always generated by the $i=0$ term of expression (29). Moreover, the strongest reason for neglecting the very small contributions of the $i>0$ terms of expression (29) is that for all values of $U / t_{1}$ all terms of the correlation-function asymptotic expansion obtained by conformal-field theory are generated by the $i=0$ term of that expression. Thus, the low-energy expression needed for our studies is of the form

$$
B_{\mathcal{N}}^{l}(k, \omega)=\sum_{\left\{\Delta J_{\alpha}^{F}\right\}} B^{l, 0}(k, \omega),
$$

where the $\sum_{\left\{\Delta J_{\alpha}^{F}\right\}}$ summation is over the J-CPHS subspaces contained in the low-energy sector of the CPHS subspace specific to the $\mathcal{N}$-electron spectral function $B_{\mathcal{N}}^{l}(k, \omega)$. Here $B^{l, 0}(k, \omega)$ is the function is given in Eq. (44) of Ref. [14] and Eq. (68) of Ref. [15] for $i=0$.

It follows from the form of the spectral-function expression (30) that the corresponding general $\mathcal{N}$-electron correlation function (2) can be written as

$$
\chi_{\mathcal{N}}^{l}(k, \omega)=l \sum_{\left\{\Delta J_{\alpha}^{F}\right\}} \int_{-\infty}^{+\infty} d \omega^{\prime} \frac{B^{l, 0}\left(k, \omega^{\prime}\right)}{\omega-\omega^{\prime}+i l 0} .
$$

Thus, the function $\tilde{\chi}_{\mathcal{N}}^{l}(x, t)$ of Eq. (4) can be expressed in terms of the double Fourier transform relative to $k$ and $\omega$ of the set of functions $B^{l, 0}(k, \omega)$ corresponding to each J-CPHS subspace, 
$\tilde{B}^{l, 0}(x, t)$, as follows

$$
\tilde{\chi}_{\mathcal{N}}^{l}(x, t)=-i 2 \pi \theta(l t) \sum_{\left\{\Delta J_{\alpha}^{F}\right\}} \tilde{B}^{l, 0}(x, t) .
$$

We start by deriving the asymptotic expression of $\tilde{B}^{l, 0}(x, t)$ associated with the low-energy behavior of $B^{l, 0}(k, \omega)$. Within the low-energy limit considered here, the second expression of Eq. (68) of Ref. [15] for $B^{l, 0}(k, \omega)$ simplifies. Indeed, the low-energy correlation-function excitation CPHS ensemble subspace is always a dominant CPHS ensemble subspace, such that the corresponding real positive coefficient $G_{C}$ defined in the unnumbered equation below Eq. (68) of Ref. [15] reads $G_{C}=1$ for the whole parameter space and thus $C_{s}=\mathcal{C}_{s}$ in Eq. (68) of that reference. The absence of independent $-1 / 2$ holons and $-1 / 2$ spinons [14,15], including of $-1 / 2$ Yang holons and $-1 / 2$ HL spinons, then implies that,

$$
\left(\prod_{\alpha=c, s} \frac{1}{C_{\alpha}}\right)=1
$$

in that general expression for $B^{l, 0}(k, \omega)$ and,

$$
l \sum_{\alpha=c, s} P_{\alpha}=l \sum_{\alpha=c, s} E_{\alpha}=0,
$$

in the argument of its function $B_{Q_{c 0}}^{l, 0} \equiv B_{Q_{c}}^{l, 0}$. Moreover, according to Eq. (7) there are no $c v$ pseudofermions and $s v^{\prime}$ pseudofermions belonging to branches such that $v>0$ and $v^{\prime}>1$, respectively, in the excited energy eigenstates which span the low-energy subspace. Thus, there are only finite occupancies for the $c \equiv c 0$ and $s \equiv s 1$ pseudofermion branches. Furthermore, there are no finite-energy $c$ and $s$ processes called processes (A) in Ref. [14] so that the number $\mathcal{N}_{\alpha \nu} \equiv \mathcal{N}_{\alpha}$ given in Eq. (61) of Ref. [15] vanishes. For all these reasons the factor

$$
\begin{aligned}
& \left(\prod_{j=1}^{D}\left(\frac{1}{N_{a}}\right)^{\mathcal{N}_{\alpha v_{j}}}\left[\sum_{\left.\mathrm{J}-\mathrm{CPHS}-\alpha v_{j}-\mathrm{A}\right)}\right]\right) \\
& =\prod_{j=1}^{2}\left(\frac{1}{N_{a}}\right)^{\mathcal{N}_{\alpha v_{j}}}=\prod_{\alpha=c, s}\left(\frac{1}{N_{a}}\right)^{\mathcal{N}_{\alpha}}=\prod_{\alpha=c, s}\left(\frac{1}{N_{a}}\right)^{0}=1,
\end{aligned}
$$

in the second expression of Eq. (68) of Ref. [15] reduces to the unity and the values

$$
l \sum_{j=1}^{D} \Delta P_{\alpha v_{j}}=l \sum_{j=1}^{2} \Delta P_{\alpha v_{j}}=l\left[\Delta P_{c}^{F}+\Delta P_{s}^{F}\right]=l k_{0}^{F}, \quad l \sum_{j=1}^{D} \Delta E_{\alpha v_{j}}=0,
$$

must be used in the argument of the function $B_{Q_{c 0}}^{l, 0} \equiv B_{Q_{c}}^{l, 0}$ appearing in the same general expression. Note that the momentum $k_{0}^{F}$ of Eq. (36) is that given in Eq. (20) and $\Delta P_{c}^{F}$ and $\Delta P_{s}^{F}$ are provided in Eq. (21).

As a result of the above simplifications, in the low-energy limit considered in this paper the second expression of Eq. (68) of Ref. [15] for $B^{l, 0}(k, \omega)$ leads to

$$
B^{l, 0}(k, \omega)=\frac{1}{2 \pi} \int_{-\infty}^{+\infty} d k^{\prime} \int_{-\infty}^{+\infty} d \omega^{\prime} B_{Q_{c}}^{l, 0}\left(k-l \Delta P_{c}^{F}-k^{\prime}-l \Delta P_{s}^{F}, \omega-\omega^{\prime}\right) B_{Q_{s}}^{l, 0}\left(k^{\prime}, \omega^{\prime}\right) .
$$


This expression can be rewritten as

$$
B^{l, 0}(k, \omega)=\frac{1}{2 \pi} \int_{-\infty}^{+\infty} d k^{\prime} \int_{-\infty}^{+\infty} d \omega^{\prime} B_{Q_{c}}^{l, 0}\left(k-l \Delta P_{c}^{F}-k^{\prime}, \omega-\omega^{\prime}\right) B_{Q_{s}}^{l, 0}\left(k^{\prime}-l \Delta P_{s}^{F}, \omega^{\prime}\right),
$$

where the function $B_{Q_{\alpha}}^{l, 0}\left(k-l \Delta P_{\alpha}^{F}, \omega\right)$ is such that

$$
\begin{aligned}
B_{Q_{\alpha}}^{l, 0}\left(k-l \Delta P_{\alpha}^{F}, \omega\right)= & \frac{N_{a}}{4 \pi} \int_{-\infty}^{+\infty} d k^{\prime} \int_{-\infty}^{+\infty} d \omega^{\prime} B_{Q_{\alpha}}^{l,+1,0}\left(k-l q_{F \alpha}^{0} \Delta N_{\alpha,+1}^{F}-k^{\prime}, \omega-\omega^{\prime}\right) \\
& \times B_{Q_{\alpha}}^{l,-1,0}\left(k^{\prime}+l q_{F \alpha}^{0} \Delta N_{\alpha,-1}^{F}, \omega^{\prime}\right), \quad \alpha=c, s .
\end{aligned}
$$

Here $B_{Q_{\alpha}}^{l, 0}\left(k^{\prime}, \omega^{\prime}\right)$ and $B_{Q_{\alpha}}^{l, \iota, 0}\left(k^{\prime}, \omega^{\prime}\right)$ are the functions defined in Eqs. (45) and (47) of Ref. [14], respectively.

For small finite values of $l \omega^{\prime}$ we use for the function $B_{Q_{\alpha}}^{l, l, 0}\left(k^{\prime}, \omega^{\prime}\right)$ the expression provided in Eq. (58) of Ref. [14]. If one uses continuum values of $k^{\prime}$, the latter expression can be rewritten as

$$
B_{Q_{\alpha}}^{l, \iota, 0}\left(k^{\prime}, \omega^{\prime}\right)=\theta\left(l \omega^{\prime}\right) \frac{2 \pi \Lambda_{\alpha, \iota}}{\sqrt{N_{a}} v_{\alpha} \Gamma\left(2 \Delta_{\alpha}^{\iota}\right)}\left(\frac{l \omega^{\prime}}{v_{\alpha}}\right)^{2 \Delta_{\alpha}^{\iota}-1} \delta\left(k^{\prime}-\frac{\iota \omega^{\prime}}{v_{\alpha}}\right), \quad \alpha=c, s, \iota= \pm 1,
$$

where $\Gamma(y)$ is the usual $\Gamma$ function, $2 \Delta_{\alpha}^{\iota}$ is the functional given in Eq. (25), $v_{\alpha}$ is the velocity provided in Eq. (28), and $\Lambda_{\alpha, \iota}$ reads

$$
\Lambda_{\alpha, \iota}=\left(\frac{N_{a}}{2 \pi}\right)^{2 \Delta_{\alpha}^{\iota}} \frac{A_{\alpha, \iota}^{(0,0)}}{\sqrt{N_{a}}}=\frac{\sqrt{S_{\alpha}^{0}} f_{\alpha, \iota}}{\left(2 \pi S_{\alpha}^{0}\right)^{2 \Delta_{\alpha}^{\iota}}}, \quad \alpha=c, s, \iota= \pm 1 .
$$

It is convenient to introduce the related quantity

$$
\Lambda_{\alpha}=\prod_{\iota} \Lambda_{\alpha, \iota}=\left(\frac{N_{a}}{2 \pi}\right)^{\left[2 \Delta_{\alpha}^{+1}+2 \Delta_{\alpha}^{-1}\right]} \frac{A_{\alpha}^{(0,0)}}{\sqrt{N_{a}}}, \quad \alpha=c, s .
$$

Here $A_{\alpha, l}^{(0,0)}$ is given in Eq. (49) of Ref. [14] and refers to the four $\alpha, \iota$ weights such that the two associated $\alpha$ pseudofermion spectral-function lowest-peak weights

$$
A_{\alpha}^{(0,0)}=\prod_{\iota} A_{\alpha, \imath}^{(0,0)}, \quad \alpha=c, s,
$$

can be expressed in terms of the $\alpha$ pseudofermion overall phase shifts (16) as follows [14]

$$
\begin{aligned}
A_{\alpha}^{(0,0)}= & A_{\alpha}^{(0,0)}\left(\Delta N_{c}, \Delta N_{s}, \Delta J_{c}^{F}, \Delta J_{s}^{F}\right) \\
= & \left(\frac{1}{N_{\alpha}^{*}}\right)^{2\left[N_{\alpha}^{0}+\Delta N_{\alpha}\right]} \prod_{q_{j} \in \mathcal{F}} \sin ^{2}\left(Q_{\alpha}\left(q_{j}\right) / 2\right) \prod_{j=1}^{N_{\alpha}^{*}-1}\left[\sin \left(\frac{\pi j}{N_{\alpha}^{*}}\right)\right]^{2\left[N_{\alpha}^{*}-j\right]} \\
& \times \prod_{q_{i} \in \mathcal{F}} \prod_{q_{j} \in \mathcal{F}} \theta\left(q_{j}-q_{i}\right) \sin ^{2}\left(\frac{Q_{\alpha}\left(q_{j}\right) / 2-Q_{\alpha}\left(q_{i}\right) / 2+\pi(j-i)}{N_{\alpha}^{*}}\right) \\
& \times \prod_{q_{i} \in \mathcal{F}} \prod_{q_{j} \in \mathcal{F}} \frac{1}{\sin ^{2}\left(\frac{\pi(j-i)+Q_{\alpha}\left(q_{j}\right) / 2}{N_{\alpha}^{*}}\right)}, \quad \alpha=c, s .
\end{aligned}
$$


In this expression $\Delta N_{\alpha}$ is the number deviation of Eq. (8), $N_{\alpha}^{*}$ is given in Eq. (13), $Q_{\alpha}\left(q_{j}\right) / 2$ is the overall phase shift (16), and $q_{j} \in \mathcal{F}$ corresponds to the set of discrete bare-momentum values in the range $q_{F \alpha,-1} \leqslant q_{j} \leqslant q_{F \alpha,+1}$ where $q_{F \alpha, \iota}$ is the J-ground state Fermi point given in Eq. (17). We emphasize that the $N_{a}$ dependence of $A_{\alpha}^{(0,0)}$ is such that the quantity $\Lambda_{\alpha}$ given in Eq. (42) is independent of $N_{a}$. Indeed, by use of the second expression of Eq. (41) such a quantity can be rewritten as

$$
\Lambda_{\alpha}=S_{\alpha}^{0} \prod_{\alpha} \frac{f_{\alpha, \iota}}{\left(2 \pi S_{\alpha}^{0}\right)^{2 \Delta_{\alpha}^{\iota}}}, \quad \alpha=c, s,
$$

where here and in that equation the related quantities $f_{\alpha, \iota}$ and $S_{\alpha}^{0}$ are those of Eqs. (49) and (50) of Ref. [14]. All quantities on the right-hand side of this equations are independent of $N_{a}$ [14].

It is straightforward to confirm that the convolution of Eq. (39) leads to the first expression of Eq. (58) of Ref. [14] for the function $B_{Q_{\alpha}}^{l, 0}(k, \omega)$ if one uses the expression (40) for the function $B_{Q_{\alpha}}^{l, \iota, 0}\left(k^{\prime}, \omega^{\prime}\right)$ in that convolution.

In contrast to the small-momentum and low-energy $\alpha$ pseudofermion particle-hole processes (C), the processes (B) create active $\alpha$ pseudofermion and/or $\alpha$ pseudofermion hole scattering centers. The active scattering centers created by these processes are those which lead to the value of the scattering part of the $\alpha$ overall phase shift given in Eq. (16). We recall that the latter value is the same for all energy eigenstates which span a given J-CPHS subspace. Thus, the general expression for the weight $A_{\alpha}^{(0,0)}$ given in Eq. (44) has also the same value for all energy eigenstates which span such a subspace. The same occurs for the overall phase shift (16), functional (25), and related weights $A_{\alpha, l}^{(0,0)}$. Hence, the quantities $\Lambda_{\alpha, \iota}$ and $\Lambda_{\alpha}$ given in Eqs. (41) and (42), respectively, have also the same value for all energy eigenstates which span a given J-CPHS subspace. It follows that the expression of each of the functions $B^{l, 0}(k, \omega)$ on the righthand side of Eq. (30) and that of each of the corresponding functions $\tilde{B}^{l, 0}(x, t)$ of Eq. (32), which we derive below, involve a single value of the two parameters $\Lambda_{\alpha}$ and four quantities $2 \Delta_{\alpha}^{\iota}$.

Since the functions of Eqs. (38) and (39) are given by convolutions of other functions, the use of the Convolution Theorem of Fourier transforms implies that the asymptotic expression of the function $\tilde{B}^{l, 0}(x, t)$ on the right-hand side of Eq. (32) can written as

$$
\tilde{B}^{l, 0}(x, t)=\left(\frac{N_{a}}{2}\right)^{2} \prod_{\alpha} \prod_{l} \tilde{B}_{Q_{\alpha}}^{l, l, 0}(x, t) .
$$

Here $\tilde{B}_{Q_{\alpha}}^{l, \iota, 0}(x, t)$ is the double Fourier transform of $B_{Q_{\alpha}}^{l, \iota, 0}\left(k^{\prime}-l \iota q_{F \alpha}^{0} \Delta N_{\alpha, l}^{F}, \omega^{\prime}\right)$. Due to the $\delta$ function in expression (40), one of the integrals involved in the derivation of such a transform is straightforward to perform, what leads to

$$
\begin{aligned}
& \tilde{B}_{Q_{\alpha}}^{l, \iota, 0}(x, t)=l e^{i l \iota q_{F \alpha}^{0} \Delta N_{\alpha, \iota}^{F} x} \frac{\Lambda_{\alpha, \iota}}{\sqrt{N_{a}}\left(v_{\alpha}\right)^{2 \Delta_{\alpha}^{\iota} \Gamma\left(2 \Delta_{\alpha}^{\iota}\right)}} \int_{0}^{\infty} d z e^{i \frac{l \iota z}{v_{\alpha}}\left(x-\iota v_{\alpha} t+i \iota 0\right)}(z)^{2 \Delta_{\alpha}^{\iota}-1}, \\
& \alpha=c, s, \iota= \pm 1 .
\end{aligned}
$$

After performing the $z$ integration of this expression one finds

$$
\tilde{B}_{Q_{\alpha}}^{l, \iota, 0}(x, t)=l e^{i l \iota q_{F \alpha}^{0} \Delta N_{\alpha, \iota}^{F} x} e^{-i l \iota \frac{\pi}{2} 2 \Delta_{\alpha}^{\iota}} \frac{\Lambda_{\alpha, \iota}}{\sqrt{N_{a}}}\left(\frac{1}{x-\iota v_{\alpha} t+i \iota 0}\right)^{2 \Delta_{\alpha}^{\iota}}, \quad \alpha=c, s, \iota= \pm 1 .
$$


In the expressions given in Eqs. (47) and (48) we have added a small infinitesimal number, $i \iota 0$, to $\left(x-\iota v_{\alpha} t\right)$. Such a number introduces the correct regularization.

By use of Eq. (48) in Eq. (46) we arrive to

$$
\tilde{B}^{l, 0}(x, t)=i \frac{e^{i l k_{0}^{F} x}}{2 \pi} \chi_{0} \prod_{\alpha} \prod_{\iota}\left(\frac{1}{x-\iota v_{\alpha} t+i \iota 0}\right)^{2 \Delta_{\alpha}^{\iota}},
$$

where the pre-factor $\chi_{0}$ is given by

$$
\chi_{0}=\chi_{0}\left(\Delta N_{c}, \Delta N_{s}, \Delta J_{c}^{F}, \Delta J_{s}^{F}\right)=-i \frac{\pi}{2} e^{-i \frac{\pi}{2} \lambda_{l}} \prod_{\alpha} \Lambda_{\alpha},
$$

and

$$
\lambda_{l}=l \sum_{\alpha} \sum_{\iota} l 2 \Delta_{\alpha}^{\iota}
$$

Finally, use of expression (49) in Eq. (32) leads to the following general expression for the correlation-function asymptotic expansion

$$
\tilde{\chi}_{\mathcal{N}}^{l}(x, t)=\theta(l t) \sum_{\left\{\Delta J_{\alpha}^{F}\right\}}\left\{e^{i l k_{0}^{F} x} \chi_{0} \prod_{\alpha} \prod_{\iota}\left(\frac{1}{x-\iota v_{\alpha} t+i \iota 0}\right)^{2 \Delta_{\alpha}^{\iota}}\right\} .
$$

In Appendix $\mathrm{A}$ it is shown that in the present low-energy limit the square of the shift in the value of the $\alpha, \iota$ pseudofermion Fermi-point $2 \Delta_{\alpha}^{\iota}$ given in Eq. (25) is the conformal dimension of the $\alpha, \iota$ primary field of conformal-field theory. In the ensuing section we clarify the relation of that field to the $\alpha$ pseudofermion operators. The asymptotic expression (52) has the same general form as that provided by conformal-field theory. Thus, that in the low-energy limit considered here the square of the shift in the value of the $\alpha, \iota$ pseudofermion Fermi-point, $2 \Delta_{\alpha}^{\iota}$, is the conformal dimension of a $\alpha, \iota$ primary field confirms that the expansion (52) coincides with the general conformal-field theory correlation-function asymptotic expansion used in the studies of Refs. [25-29,34]. However, we emphasize that within the PDT the important functional (24) is well defined for all energy scales and corresponds to a much more general paradigm [14], and thus such a connection only emerges in the low-energy limit considered here.

The Fourier transforms considered above used low-energy expressions which capture the universal part of the asymptotic expansion of correlation functions, Eq. (52). In this paper we do not study the corresponding logarithmic corrections, which are specific to each correlation function [22].

\section{Pre-factors universal form and relation of the low-energy physics to the scattering properties at all energy scales}

The main result of the previous section is the evaluation of the pre-factors $\chi_{0}$, Eq. (50), of each term of the correlation-function asymptotic expansion (52). Here we discuss the universal character of such pre-factors and their relation to the scattering properties at all energy scales. In addition, we address other issues such as the qualitative difference between some of the spectral features of the low-energy and finite-energy metallic phases and the relation of the low-energy pseudofermion description to the conformal-field theory primary fields and Virasoro-algebra generators [24]. 


\subsection{Pre-factors and relation to the general scattering properties}

The universal character of the asymptotic expansion (52) is such that the value of the conformal dimensions $2 \Delta_{\alpha}^{\iota}=2 \Delta_{\alpha}^{\iota}\left(\Delta N_{c}, \Delta N_{s}, \Delta J_{c}^{F}, \Delta J_{s}^{F}\right)$ defined in Eq. (25) only depends on the specific correlation function through the values of the four deviations $\Delta N_{c}, \Delta N_{s}, \Delta J_{c}^{F}$, and $\Delta J_{s}^{F}$ of each allowed excitation J-subspace. Otherwise, the $U / t_{1}, n$, and $m$ dependence of the two-pseudofermion phase-shift parameters $\xi_{\alpha \alpha^{\prime}}^{j}$ of Eq. (25) is specific to the model but is the same for all its correlation functions.

Importantly, careful analysis of the form of the quantities defined in Eqs. (42) and (44) reveals that the same occurs for the pre-factors $\chi_{0}$ of the correlation-function asymptotic expansion. Indeed, the expression of the associated weight of Eq. (44) involves the overall phase shift $Q_{\alpha}(q) / 2$ given in Eqs. (16) and (22), which for each value of $q$ also depends on the specific correlation function through the values of the four deviation numbers $\Delta N_{c}, \Delta N_{s}, \Delta J_{c}^{F}$, and $\Delta J_{s}^{F}$ of each allowed J-subspace. Moreover, the $q_{j}$ and $q_{i}$ products of the $A_{\alpha}^{(0,0)}$ expression given in Eq. (44) run over the ranges $q_{F \alpha,-1} \leqslant q_{j} \leqslant q_{F \alpha,+1}$ and $q_{F \alpha,-1} \leqslant q_{i} \leqslant q_{F \alpha,+1}$, respectively, with the limiting values $q_{F \alpha, \pm 1}$, Eq. (17), involving the deviation given in Eq. (18), whose value is solely determined by the deviations $\Delta N_{\alpha}$ and $\Delta J_{\alpha}^{F}$. Thus, the pre-factors $\chi_{0}=\chi_{0}\left(\Delta N_{c}, \Delta N_{s}, \Delta J_{c}^{F}, \Delta J_{s}^{F}\right)$ value also depends on the specific correlation function through the values of the four deviation numbers $\Delta N_{c}, \Delta N_{s}, \Delta J_{c}^{F}$, and $\Delta J_{s}^{F}$ only, as the conformal dimensions. Otherwise, the $U / t_{1}$, $n$, and $m$ dependence of the two-pseudofermion phase shifts involved in the $\chi_{0}$ expression is specific to the model but is again the same for all its correlation functions. Such two-pseudofermion phase shifts are defined by Eqs. (A.1)-(A.7) of Appendix A.

The form of the pre-factors $\chi_{0}$ of all terms of the asymptotic expansion (52) is universal for all correlation functions and is given in Eq. (50). Their value is controlled by the overall pseudofermion and hole phase shifts, Eq. (16), through the dependence on these shifts of the two weights $A_{\alpha}^{(0,0)}$ of Eq. (44) and four functionals $2 \Delta_{\alpha}^{\iota}$ of Eqs. (24) and (25). Concerning the relation of the latter quantities to the scattering mechanisms, note that in the $A_{\alpha}^{(0,0)}$ expression (44) the bare-momentum products run over the overall phase shifts of the $\alpha$ pseudofermion scatterers with bare momentum inside the J-ground-state Fermi sea, whose scattering centers are the $c$ and $s$ pseudofermion and holes created at the J-ground-state Fermi points by the elementary processes (B). Furthermore, the four conformal dimensions of the primary fields equal the square of the shifts in the two $c$ and two $s$ pseudofermion canonical-momentum Fermi points. The four functionals $2 \Delta_{\alpha}^{\iota}$ and the two weights $A_{\alpha}^{(0,0)}$ also play an important role in the finite-energy scattering properties, by controlling the unusual spectral properties of the model [14,21] and real materials $[16,18]$ at all energy scales. Thus, our results reveal the connection of the low-energy quantities to the scattering mechanisms that control the spectral properties at all energy scales.

\subsection{The low-energy TLL and finite-energy metallic phases}

The above discussed connection of the low-energy quantities to the scattering mechanisms that following the PDT studies of Refs. $[14,15]$ control the spectral properties at all energy scales can be used to clarify an interesting issue related to different behaviors of the low-energy and finite-energy spectral-weight distributions. Indeed, there are some features in the spectral functions (3) whose $k$ and $\omega$ dependence is qualitatively different for small values of $\omega$, where the low-energy TLL behavior dominates, and for finite $\omega$ values. Here we address such an issue, whose understanding involves general mechanisms already studied in Ref. [14]. 
Due to the convolutions in Eqs. (38) and (39), the asymptotic expansion (52) was obtained by use in expression (46) of the $\alpha, \iota$ spectral function (40). To study the above issue it is useful to perform the inverse Fourier transform of the asymptotic expansion (52) relative to both $x$ and $t$. This provides the behavior of $\chi_{\mathcal{N}}^{l}(k, \omega)$ near the singularities corresponding to the branch lines whose linear shape is defined by the following parametric equations

$$
\omega=\iota v_{\alpha}\left(k-l k_{0}^{F}\right), \quad \alpha=c, s, \iota= \pm 1,
$$

where the momentum $k_{0}^{F}$ and velocity $v_{\alpha}$ are given in Eqs. (20) and (28), respectively. The obtained expression corresponds to a range of small values of $\omega$ and $\left(k-l k_{0}^{F}\right)$ such that $\omega \approx \imath v_{\alpha}\left(k-l k_{0}^{F}\right)$. By performing the double inverse Fourier transform relative to $x$ and $t$ of the leading-order term of the general asymptotic expansion (52), one finds that this behavior is associated with the following $k$ and $\omega$ dependence of the correlation function (2)

$$
\chi_{\mathcal{N}}^{l}(k, \omega) \propto\left(l \omega-\iota v_{\alpha}\left(k-l k_{0}^{F}\right)\right)^{\zeta \alpha, \iota}, \quad \alpha=c, s, \iota= \pm 1,
$$

where the exponent reads

$$
\zeta_{\alpha, \iota}=-1+2 \Delta_{\alpha}^{\iota}+2 \Delta_{\bar{\alpha}}^{+1}+2 \Delta_{\bar{\alpha}}^{-1}, \quad \alpha=c, s, \iota= \pm 1,
$$

and $2 \Delta_{\alpha}^{\iota}$ is the functional given in Eq. (25), $\bar{c}=s$, and $\bar{s}=c$. For the values of $k$ and $\omega$ that these expressions refer to, the real and imaginary parts of $\chi_{\mathcal{N}}^{l}(k, \omega)$ have the same $k$ and $\omega$ dependence, but differ in the pre-factors. Thus, one also finds

$$
B_{\mathcal{N}^{(}}^{l}(k, \omega) \propto\left(l \omega-\iota v_{\alpha}\left(k-l k_{0}^{F}\right)\right)^{\zeta_{\alpha, \iota}}, \quad \alpha=c, s, \iota= \pm 1,
$$

for the general spectral function given in Eq. (3).

When applied to specific $\mathcal{N}$-electron spectral functions, expression (56) with the power-law exponent given in Eq. (55) provides the universal and well, known low-energy TLL behavior for the 1D Hubbard model [25-29,34], Tomonaga-Luttinger model [45-47], and many other models whose low-energy physics corresponds to the same universality class. When $\zeta_{\alpha, \iota}<0$, such an expression refers to a linear singular spectral feature.

The PDT studies of Ref. [14] reveal that the spectral feature whose shape is defined by Eq. (53) is the low-energy part of a spectral-function $\alpha$ branch line which also exists for finite energy values. The parametric equations which define the $(k, \omega)$-plane points belonging to such a $\alpha$ pseudofermion (or $\alpha$ pseudofermion hole) branch line is of the general form

$$
k=l\left[k_{0}^{F}-c_{1} \iota q_{F \alpha}^{0}+c_{1} q\right], \quad \omega=l E_{\alpha}(k)=l c_{1} \epsilon_{\alpha}(q), \quad \alpha v=c, s, \iota= \pm 1,
$$

where

$$
q \in \begin{cases}{\left[-q_{F \alpha}^{0},+q_{F \alpha}^{0}\right],} & \alpha=c, s, \iota= \pm 1, c_{1}=-1, \\ {\left[+q_{F \alpha}^{0},+q_{\alpha}^{0}\right],} & \alpha=c, s, \iota=+1, c_{1}=+1, \\ {\left[-q_{\alpha}^{0},-q_{F \alpha}^{0}\right],} & \alpha=c, s, \iota=-1, c_{1}=+1,\end{cases}
$$

and the constant $c_{1}$ is such that $c_{1}=+1$ (and $c_{1}=-1$ ) for creation of a $\alpha$ pseudofermion (and a $\alpha$ pseudofermion hole), as discussed below. Note that for

$$
q=\iota q_{F \alpha}^{0}+l c_{1}\left(k-l k_{0}^{F}\right), \quad \alpha=c, s, \iota= \pm 1,
$$

with $\left(k-l k_{0}^{F}\right)$ small one finds

$$
l E_{\alpha}(k)=l c_{1} \epsilon_{\alpha}\left(\iota q_{F \alpha}^{0}+l c_{1}\left(k-l k_{0}^{F}\right)\right) \approx \iota v_{\alpha}\left(k-l k_{0}^{F}\right), \quad \alpha=c, s, \iota= \pm 1 .
$$


Here we used the property that $\epsilon_{\alpha}\left(\iota q_{F \alpha}^{0}\right)=0[14,20,42,48]$. This confirms that for baremomentum values $q$ in the vicinity of $\iota q_{F \alpha}^{0}$ the energy $\omega$ is small and the line defined by the parametric equation (57) becomes indeed the line (53). However, although the latter line is continuously reached from the general line (57) as $q$ approaches $\iota q_{F \alpha}^{0}$, the $k$ and $\omega$ dependence of the corresponding spectral feature has two regimens, for small and finite values of $\omega$, respectively.

Indeed, use of the general PDT reveals that the line defined by the parametric equation (57) corresponds to a spectral feature called $\alpha$ pseudofermion $\left(c_{1}=+1\right)$ or $\alpha$ pseudofermion hole $\left(c_{1}=-1\right)$ branch line [14]. Such spectral features were observed for the one-electron removal case by photoemission experiments in quasi-1D compounds [11,16]. A spectral-function $\alpha$ branch line is produced by creation for the values of the momentum and energy given in Eq. (57) of a $\alpha$ pseudofermion or $\alpha$ pseudofermion hole, as a result of ground-state-excited-energyeigenstate transitions with such values for the excitation momentum and energy. Therefore, the branch lines are named according to the corresponding pseudofermion or pseudofermion hole, once the shape of the branch line in the $(k, \omega)$-plane coincides with that of that object energy dispersion.

The use of the spectral-function expressions derived in Ref. [14] reveals that for $(k, \omega)$-plane points located just above $(l=+1)$ or below $(l=-1)$ the branch line whose shape is defined in Eq. (57), the weight distribution has the following form for finite values of $\omega$,

$$
B_{\mathcal{N}}^{l}(k, \omega) \propto\left(l \omega-E_{\alpha}(k)\right)^{\zeta_{\alpha}(k)}, \quad \alpha=c, s,
$$

where the exponent reads

$$
\zeta_{\alpha}(k)=-1+2 \Delta_{c}^{+1}(k)+2 \Delta_{s}^{-1}(k)+2 \Delta_{c}^{+1}(k)+2 \Delta_{s}^{-1}(k), \quad \alpha=c, s .
$$

In this expression the parameters $2 \Delta_{\alpha}^{\iota}(k)$ correspond to the general functional given in Eq. (24). However, they are not given by expression (25), which corresponds to the low-energy limit of such functionals. In the present general case the phase-shift dependence is that provided in Eq. (40) of Ref. [14]. The dependence on the momentum $k$ occurs through the corresponding dependence on the scattering center bare-momentum of the phase-shift scattering component given in Eq. (36) of that reference. In contrast to the low-energy limit studied here, the general PDT expressions derived in Ref. [14] include contributions from pseudofermion and/or hole scattering centers created off the Fermi points for finite values of the excitation energy. The above $\alpha$ pseudofermion or hole which generates the spectral feature (61) is an example of such scattering centers. Note that when the exponent (62) is such that $\zeta_{\alpha}(k)<0$, expression (61) refers to a singular spectral feature.

As $k \rightarrow l k_{F}^{0}$ (and $q \rightarrow \iota q_{F \alpha}^{0}$ ) and $\omega \rightarrow 0$, the parameters $2 \Delta_{\alpha}^{\iota}(k)$ of the exponent expression (62) become those of Eqs. (25) and (55), with $2 \Delta_{\alpha}^{\iota}\left(l k_{0}^{F}\right)=2 \Delta_{\alpha}^{\iota}$. This result together with comparison of the $2 \Delta_{\alpha}^{\iota}$ dependence of the exponents (55) and (62) confirms that the latter exponent does not evolve continuously onto the former exponent as $q \rightarrow \iota q_{F \alpha}^{0}$ and $\omega \rightarrow 0$. The origin of such two different behaviors of the spectral function in the vicinity of the branch line for small and finite values of $\omega$, respectively, can be explained by an effect which is as a particular case of a general PDT mechanism studied in Ref. [14]. As $q \rightarrow \iota q_{F \alpha}^{0}$ and thus $\omega \rightarrow 0$ the spectral function corresponds to the vicinity of a $\alpha \nu=c, s$ branch line end point, $\left(k=l k_{0}^{F}, \omega=0\right)$. That for this low-energy TLL limit the expression of the spectral function in the vicinity of the $c$ or $s$ branch-line is not that of Eq. (61) results from a resonance effect: the branch line group velocity $v_{\alpha}(q)$ equals the velocity $v_{\alpha}\left(\iota q_{F \alpha}^{0}\right)=\iota v_{\alpha}$ associated with the $\alpha, \iota$ pseudofermion particle-hole excitation sub-branch generated by the elementary processes $(C)$. Due to such a resonance effect, which also occurs for finite energies corresponding to the lower limits of the first, second, 
and higher-order upper Hubbard bands, it is shown in Ref. [14] that the momentum and energy dependence of the spectral function in the vicinity of the $\alpha$ pseudofermion or pseudofermion hole branch line is instead given by Eq. (73) of that reference. The above low-energy expression (54) corresponds to a particular case of the general expression given in that equation. In turn, the finite- $\omega$ expression (61) is a particular case of the general expression (70) of that reference for the spectral function in the vicinity of $c$ and $s$ pseudofermion branches lines considered here. The latter expression corresponds to the same spectral function in the vicinity of any pseudofermion branch line, including those corresponding to the $c v$ and $s v^{\prime}$ pseudofermion branches such that $v>0$ and $\nu^{\prime}>1$, respectively.

We note that there is an intermediate regimen in the vicinity of the $\alpha$ branch where the spectral function is neither given by the low-energy TLL expression (54) nor by the finite-energy expression (61). These expressions correspond to $v_{\alpha}(q) \approx \iota v_{\alpha}$ and $v_{\alpha}(q) \neq \iota v_{\alpha}$, respectively. The energy and momentum widths of the crossover regimen are infinitesimal. In turn, the energy and momentum widths of the low-energy linear regimen of Eqs. (53) and (56) are controlled by the value of $\left|v_{\alpha}(q)-\iota v_{\alpha}\right|$. The low-energy TLL behavior emerges when such difference can be written as

$$
\left|v_{\alpha}(q)-\imath v_{\alpha}\right| \approx\left|a_{\alpha}\left(q_{F \alpha}^{0}\right)\left(k-l k_{0}^{F}\right)\right|, \quad a_{\alpha}(q)=\frac{\partial v_{\alpha}(q)}{\partial q}, \quad \alpha=c, s, \iota= \pm 1,
$$

where the $q$ values are in the ranges given in Eq. (58) and the relation between $k$ and $q$ is defined by the first expression of Eq. (57). As the value of $q$ approaches $\iota q_{F \alpha}^{0}$ the behavior (63) is reached.

For smaller values of $\left|a_{\alpha}\left(q_{F \alpha}^{0}\right)\right|$ the value of $\left|v_{\alpha}(q)-\iota v_{\alpha}\right|$ can remain small for larger values of $\left|\left(k-l k_{0}^{F}\right)\right|$ and thus of $\omega \approx \iota v_{\alpha}\left(k-l k_{0}^{F}\right)$. It follows that the momentum and energy widths of the $(k, \omega)$-plane region in the vicinity of $\left(l k_{0}^{F}, 0\right)$ where the TLL liquid behavior (54) is valid increase for decreasing values of $\left|a_{\alpha}\left(q_{F \alpha}^{0}\right)\right|$, provided that $v_{\alpha}$ is finite. For instance, in the limit of zero spin density, $m \rightarrow 0$, the value of $\left|a_{S}(q)\right|$ is small in two relatively large $q$ regions in the vicinity of $q=-k_{F}$ and $q=+k_{F}$, respectively, and thus the domain of the corresponding spin $s$ branch lines where the low-energy TLL expression (56) is valid increases in that limit.

\subsection{Relation to conformal-field theory primary fields and Virasoro algebras}

The relation of the low-energy conformal-field theory $[23,24]$ to bosonization $[1,22]$ is well established. Thus, here we briefly discuss the connection of the general pseudofermion description to the conformal-field theory primary fields and Virasoro-algebra generators $[23,24,43]$. Implicitly, that also provides information about the relation of that description to bosonization.

In the limit of low-energy considered here the reduced J-CPHS subspaces of the general PDT [14] are spanned by a single energy eigenstate. We have called it J-ground state: it is the lowestenergy state of a J-CPHS subspace. Within the pseudofermion description a J-ground state can be written as

$$
|J-\mathrm{GS}\rangle=\prod_{\alpha} \hat{U}_{\alpha}^{\dagger} \prod_{\iota} \mathcal{F}_{\alpha, \iota}|\mathrm{GS}\rangle,
$$

where the initial ground state reads

$$
|\mathrm{GS}\rangle=\prod_{\alpha} \prod_{q_{j}=-q_{F \alpha}^{0}}^{+q_{F \alpha}^{0}} f_{q_{j}, \alpha}^{\dagger}|0\rangle,
$$


and $|0\rangle$ is the pseudofermion vacuum such that $f_{q_{j}, \alpha}|0\rangle=0$. The operator $\mathcal{F}_{\alpha, \iota}^{\dagger}$ and the transpose of the operator $\hat{U}_{\alpha}^{\dagger}$ appearing in expression (64) are given by

$$
\begin{aligned}
& \mathcal{F}_{\alpha,-1}=\left[\theta\left(\Delta N_{\alpha,-1}^{0, F}\right) \prod_{q_{j}=q_{F \alpha,-1}^{0}}^{-q_{F \alpha}^{0}} f_{q_{j}, \alpha}^{\dagger}+\theta\left(-\Delta N_{\alpha,-1}^{0, F}\right) \prod_{q_{j}=-q_{F \alpha}^{0}}^{q_{F \alpha,-1}^{0}} f_{q_{j}, \alpha}\right], \quad \alpha=c, s, \\
& \mathcal{F}_{\alpha,+1}=\left[\theta\left(\Delta N_{\alpha,+1}^{0, F}\right) \prod_{q_{j}=q_{F \alpha}^{0}}^{q_{F \alpha,+1}^{0}} f_{q_{j}, \alpha}^{\dagger}+\theta\left(-\Delta N_{\alpha,+1}^{0, F}\right) \prod_{q_{j}=q_{F \alpha,+1}^{0}}^{q_{F \alpha}^{0}} f_{q_{j}, \alpha}\right], \quad \alpha=c, s,
\end{aligned}
$$

and

$$
\hat{U}_{\alpha}=\exp \left\{\sum_{j=1}^{N_{\alpha}^{*}} f_{q_{j}, \alpha}^{\dagger}\left[f_{\bar{q}_{j}, \alpha}-f_{q_{j}, \alpha}\right]\right\}, \quad \alpha=c, s,
$$

respectively. In the pseudofermion operator $f_{\bar{q}_{j}, \alpha}$ of Eq. (67) $\bar{q}_{j}=q_{j}^{e x}+Q_{\alpha}^{\Phi}\left(q_{j}\right) / L=q_{j}+$ $Q_{\alpha}\left(q_{j}\right) / L$ where $q_{j}^{e x}=q_{j}+Q_{\alpha}^{0}\left(q_{j}\right) / L$ denotes the excited-state discrete bare-momentum values. Moreover, $q_{j}$ stands for the ground-state discrete bare-momentum values and in the four pseudofermion operators of Eq. (66) and two remaining pseudofermion operators of Eq. (67) the discrete canonical-momentum values are those of the initial ground state (65) such that $Q_{\alpha}^{\Phi}\left(q_{j}\right) / 2=0$ and, therefore, $\bar{q}_{j}=q_{j}$. The operator (67) is unitary and leaves the pseudofermion vacuum invariant and thus $\hat{U}_{\alpha}^{\dagger}|0\rangle=|0\rangle$.

Once the functional $2 \Delta_{\alpha}^{\iota}$ given in Eq. (25) is shown in Appendix A to be the conformal dimension of the $\alpha, \iota$ primary field, it is straightforward to show by analysis of the corresponding finite-size energy and momentum spectra that the J-ground state (64) is a highest-weight state (HWS) of the model $c$ and $s$ Virasoro algebras [24]. Thus, the $\alpha$, ८ operator

$$
\mathcal{G}_{\alpha, \iota}=\hat{U}_{\alpha}^{\dagger} \mathcal{F}_{\alpha, \iota} \hat{U}_{\alpha}, \quad \alpha=c, s, \iota= \pm 1,
$$

where $\mathcal{F}_{\alpha, \iota}$ and $\hat{U}_{\alpha}$ are expressed in terms of $\alpha$ pseudofermion operators in Eqs. (66) and (67), respectively, refers to the pseudofermion representation of the corresponding $\alpha, \iota$ primary field. It follows that the initial ground state (65) plays the role of the vacuum of conformal-field theory and the zero-energy and finite-momentum processes (B) generate the HWSs of the $c$ and $s$ Virasoro algebras from such a vacuum.

For the pseudofermion description, application onto the ground state of the operator $\mathcal{G}_{\alpha^{\prime}, \iota}$ creates $\left|\Delta N_{\alpha^{\prime}, l}^{0, F}\right| \alpha^{\prime}$ pseudofermion scattering centers $\left(\Delta N_{\alpha^{\prime}, l}^{0, F}>0\right)$ or $\alpha^{\prime}$ pseudofermion-hole scattering centers $\left(\Delta N_{\alpha^{\prime}, \iota}^{0, F}<0\right)$ at the $\alpha^{\prime}, \iota$ Fermi point. This leads to an overall phase shift $Q_{\alpha}(q) / 2$ for all $\alpha$ pseudofermions $\left(N_{\alpha}(q)=1\right)$ or $\alpha$ pseudofermion holes $\left(N_{\alpha}(q)=0\right)$ of baremomentum $q \in\left[-q_{\alpha}^{0},+q_{\alpha}^{0}\right]$. In particular, this shifts the $\alpha$, เ canonical-momentum Fermi point by $\Delta \bar{q}_{F \alpha, \iota}=\left[\Delta q_{F \alpha, \iota}^{0}+Q_{\alpha}\left(\iota q_{F \alpha}^{0}\right) / L\right]$. The square of such a shift in units of $2 \pi / L$ is denoted by $2 \Delta_{\alpha}^{\iota}$ in Eq. (24). In the present low-energy limit, the latter quantity has the form given in Eq. (25) and for the conformal-field theory it is the conformal dimension of the $\alpha, \iota$ primary field.

On the other hand, the generators of the small-momentum and low-energy $\alpha$ pseudofermion particle-hole processes (C) in the vicinity of the $\alpha, \iota$ Fermi point, correspond in the present lowenergy limit to the generators of two $\alpha=c, s$ Virasoro algebras [24]. These generators have a much simpler form in terms of the pseudofermion creation and annihilation operators than that of 
those given in Eqs. (66)-(68). Thus, the excited energy eigenstates generated from the J-ground state by the elementary processes (C) correspond to the tower of states of conformal-field theory. A crucial point of the pseudofermion scattering theory is that the $\alpha$ pseudofermions and holes created by the latter processes are not active scattering centers. As discussed above, the overall phase shifts generated by the created pseudofermions exactly cancel those originated by creation of the corresponding holes. This implies that the overall scattering phase shift (15) has for each $\alpha$ pseudofermion or hole scatterer of momentum $q \in\left[-q_{\alpha}^{0},+q_{\alpha}^{0}\right]$ the same value given in Eq. (16) for all excited states generated by the elementary processes $(C)$ from a given J-ground state. For the conformal-field theory, this means that all tower states obtained from application of the generators of each of the two $\alpha$ Virasoro algebras onto a given HWS correspond to the same value of the conformal dimension $2 \Delta_{\alpha}^{ \pm 1}$ of the two corresponding $\alpha, \pm 1$ primary fields.

Thus, while the pseudofermion scattering controls the model spectral properties at all energy scales [14], in the limit of low energy considered in this paper the pseudofermion operators are closely related to the conformal-field theory operators and fields. This reveals that rather than corresponding to the original electrons, the conformal-field theory spectrum and operators correspond to the low-energy limit of the general pseudofermion description.

\section{Concluding remarks}

In this paper we have shown that in the limit of low energy the general finite-energy spectralfunction expressions derived in Refs. [14,15] by means of the PDT fully recover the TLL universal expressions of correlation and spectral functions. Importantly, we were able to derive explicit expressions for the pre-factors $\chi_{0}$, Eq. (50), of all terms of the asymptotic expansion (52) for the correlation functions of the 1D Hubbard model. Furthermore, we have shown that the form of these pre-factors is universal for all correlation functions.

Our results have also clarified the relation of the low-energy TLL behavior to the general scattering mechanisms which control the model exotic spectral properties at all energy scales. Such a relation was used in the description of the effects behind the qualitative difference in the momentum and energy dependence of the low-energy and finite-energy parts of important singular features of the general spectral functions given in Eq. (3). The low-energy connection of the conformal-field primary fields and Virasoro algebra generators to the pseudofermion description was also clarified.

While the studies of this paper considered the 1D Hubbard model, which describes successfully some of the exotic properties observed in low-dimensional materials [11,16-18,49], our results are of general nature for many integrable interacting problems $[1,50]$ and therefore have wide applicability. Such results provide a broader understanding of the low-energy properties of carbon nanotubes [4,5], ballistic wires [6], quasi-1D conductors [7,8], and interacting ultracold fermionic atoms in 1D optical lattices [35,37]. Indeed, our results relate these properties to the general scattering processes of the objects whose occupancy configurations describe the exotic quantum phases of matter corresponding to different energy scales of quasi-1D materials and systems. This is confirmed for finite energies in Refs. $[11,16]$, where the general PDT weight distributions $[14,15]$ are shown to describe the photoemission features of quasi-1D compounds for the whole finite-energy band width, whereas the TLL universal behavior was observed in quasi-1D materials and systems whose low-energy phase is metallic [4-9], as mentioned in Section 1. 


\section{Acknowledgements}

We thank Patrick A. Lee, Sung-Sik Lee, João M.B. Lopes dos Santos, and Tiago C. Ribeiro for stimulating discussions. J.M.P.C. and L.M.M. thank the hospitality and support of MIT and the financial support of FCT under the grant POCTI/FIS/58133/2004, J.M.P.C. and K.P. thank the support of the ESF Science Programme INSTANS 2005-2010, J.M.P.C. thanks the financial support of the Gulbenkian Foundation and Fulbright Commission, and K.P. thanks the financial support of the OTKA grant T049607.

\section{Appendix A. The $c$ and $s$ two-pseudofermion phase shifts and Fermi point shifts}

Here we define the two-pseudofermion phase shifts $\pi \Phi_{\alpha \alpha^{\prime}}\left(q, q^{\prime}\right)$ on the right-hand side of Eqs. (15) and (22) for the scattering part of the $\alpha$ overall phase shift at bare-momentum $q$, Eq. (16). Furthermore, we show that the low-energy expression (25) of the square of the four $\alpha, \iota$ canonical-momentum Fermi points in units of $2 \pi / L$ equals that of the conformal dimension of the conformal-field theory four $\alpha, \iota$ primary fields used in the studies of Refs. [25-29,34]. We start by the definition of the two-pseudofermion phase shifts. These quantities can be expressed as,

$$
\pi \Phi_{\alpha \alpha^{\prime}}\left(q, q^{\prime}\right)=\pi \bar{\Phi}_{\alpha, \alpha^{\prime}}\left(\frac{4 t_{1} \Lambda_{\alpha}^{0}(q)}{U}, \frac{4 t_{1} \Lambda_{\alpha^{\prime}}^{0}\left(q^{\prime}\right)}{U}\right), \quad \alpha, \alpha^{\prime}=c, s,
$$

where $\pi \bar{\Phi}_{\alpha, \alpha^{\prime}}\left(r, r^{\prime}\right)$ is the corresponding rapidity two-pseudofermion phase shift defined below and

$$
\Lambda_{c}^{0}(q)=\sin k^{0}(q),
$$

$k^{0}(q)$, and $\Lambda_{s}^{0}(q)$ are ground-state rapidity functions [20]. Those are single-valued functions of the bare-momentum $q$. Thus, they can be given in terms of their inverse functions, which are the functions $q_{c}^{0}(k)$ and $q_{s}^{0}(\Lambda) \equiv q_{s 1}^{0}(\Lambda)$, respectively, defined in Eq. (A.1) of Ref. [14].

The rapidity two-pseudofermion phase shifts $\pi \bar{\Phi}_{\alpha \alpha^{\prime}}\left(r, r^{\prime}\right)$ on the right-hand side of Eq. (A.1) are particular cases of the corresponding general PDT rapidity two-pseudofermion phase shifts. In spite of a different notation for the $c v$ and $s v^{\prime}$ branches of Refs. [14,15,21], such that $v=$ $\gamma$ and $\nu^{\prime}=\gamma+1$, respectively, the general integral equations which define the rapidity twopseudofermion phase shifts $\bar{\Phi}_{\alpha v, \alpha^{\prime} v^{\prime}}\left(r, r^{\prime}\right)$ are those given in Eqs. (B30)-(B40) of Ref. [48]. While the phase shifts $\pi \bar{\Phi}_{\alpha \alpha^{\prime}}\left(r, r^{\prime}\right)$ considered here refer to the two $\alpha=c, s$ pseudofermion branches whose occupancy configurations describe the low-energy eigenstates, the phase shifts $\bar{\Phi}_{\alpha \nu, \alpha^{\prime} v^{\prime}}\left(r, r^{\prime}\right)$ refer to all the pseudofermion branches. From direct use of the general system of coupled integral equations which defines the PDT two-pseudofermion phase shifts, we find that the phase shifts $\pi \bar{\Phi}_{\alpha \alpha^{\prime}}\left(r, r^{\prime}\right)$ involved in our low-energy study are uniquely defined by the following integral equations

$$
\begin{aligned}
\pi \bar{\Phi}_{s s}\left(r, r^{\prime}\right)= & \arctan \left(\frac{r-r^{\prime}}{2}\right)-\frac{1}{\pi} \int_{-r_{c}^{0}}^{r_{c}^{0}} d r^{\prime \prime} \frac{\arctan \left(r^{\prime \prime}-r^{\prime}\right)}{1+\left(r-r^{\prime \prime}\right)^{2}} \\
& +\int_{-r_{s}^{0}}^{r_{s}^{0}} d r^{\prime \prime} G\left(r, r^{\prime \prime}\right) \pi \bar{\Phi}_{s s}\left(r^{\prime \prime}, r^{\prime}\right),
\end{aligned}
$$




$$
\begin{aligned}
& \pi \bar{\Phi}_{c s}\left(r, r^{\prime}\right)=-\arctan \left(r-r^{\prime}\right)+\frac{1}{\pi} \int_{-r_{s}^{0}}^{r_{s}^{0}} d r^{\prime \prime} \frac{\pi \bar{\Phi}_{s s}\left(r^{\prime \prime}, r^{\prime}\right)}{1+\left(r-r^{\prime \prime}\right)^{2}}, \\
& \pi \bar{\Phi}_{s c}\left(r, r^{\prime}\right)=-\arctan \left(r-r^{\prime}\right)+\int_{-r_{s}^{0}}^{r_{s}^{0}} d r^{\prime \prime} G\left(r, r^{\prime \prime}\right) \pi \bar{\Phi}_{s c}\left(r^{\prime \prime}, r^{\prime}\right),
\end{aligned}
$$

and

$$
\pi \bar{\Phi}_{c c}\left(r, r^{\prime}\right)=\frac{1}{\pi} \int_{-r_{s}^{0}}^{r_{s}^{0}} d r^{\prime \prime} \frac{\pi \bar{\Phi}_{s c}\left(r^{\prime \prime}, r^{\prime}\right)}{1+\left(r-r^{\prime \prime}\right)^{2}} .
$$

In the above equations the function $\arctan (y)$ corresponds to the branch such that $-\pi / 2 \leqslant$ $\arctan (y) \leqslant+\pi / 2$, the kernel $G\left(r, r^{\prime}\right)$ is given by

$$
G\left(r, r^{\prime}\right)=-\frac{1}{2 \pi}\left[\frac{1}{1+\left[\left(r-r^{\prime}\right) / 2\right]^{2}}-\frac{2}{\pi} \int_{-r_{c}^{0}}^{r_{c}^{0}} d r^{\prime \prime} \frac{1}{\left[1+\left(r-r^{\prime \prime}\right)^{2}\right]\left[1+\left(r^{\prime}-r^{\prime \prime}\right)^{2}\right]}\right],
$$

and the integration limiting values read

$$
r_{c}^{0}=\frac{4 t_{1} \sin Q}{U}, \quad r_{s}^{0}=\frac{4 t_{1} B}{U},
$$

where $Q=k^{0}\left(2 k_{F}\right)$ and $B=\Lambda_{s}^{0}\left(k_{F \downarrow}\right)$ are the parameters appearing in the expressions of Ref. [12]. They are such that $q_{c}^{0}( \pm Q)= \pm 2 k_{F}$ and $q_{S}^{0}( \pm B)= \pm k_{F \downarrow}$, their value being selfconsistently defined by the solution of the relations given in Eq. (A.5) of Ref. [14].

Finally, let us confirm that the low-energy limit of the square of the shift in the value of the $\alpha, \iota$ canonical-momentum Fermi point given in Eq. (25) is indeed the conformal dimension of the $\alpha, \iota$ primary field. To reach such a goal, we start by noting that combination of Eqs. (26) and (A.1) reveals that the parameters defined in Eq. (26) can for $j=1$ be expressed as

$$
\xi_{\alpha \alpha^{\prime}}^{1}=\Omega_{\alpha \alpha^{\prime}}\left(r_{\alpha}^{0}\right), \quad \alpha, \alpha^{\prime}=c, s,
$$

where the function $\Omega_{\alpha \alpha^{\prime}}(r)$ is given by

$$
\Omega_{\alpha \alpha^{\prime}}(r)=\delta_{\alpha, \alpha^{\prime}}+\sum_{\iota= \pm 1} \iota \bar{\Phi}_{\alpha, \alpha^{\prime}}\left(r, \iota r_{\alpha^{\prime}}^{0}\right), \quad \alpha, \alpha^{\prime}=c, s .
$$

Based on Eqs. (A.3)-(A.7), it is straightforward to confirm that the functions defined by Eq. (A.10) obey the following integral equations:

$$
\begin{aligned}
& \Omega_{s s}(r)=1+\int_{-r_{s}^{0}}^{r_{s}^{0}} d r^{\prime \prime} G\left(r, r^{\prime \prime}\right) \Omega_{s s}\left(r^{\prime \prime}\right), \\
& \Omega_{c s}(r)=\frac{1}{\pi} \int_{-r_{s}^{0}}^{r_{s}^{0}} d r^{\prime \prime} \frac{\Omega_{s s}\left(r^{\prime \prime}\right)}{1+\left(r-r^{\prime \prime}\right)^{2}},
\end{aligned}
$$




$$
\Omega_{s c}(r)=\frac{1}{\pi}\left[\arctan \left(r+r_{c}^{0}\right)-\arctan \left(r-r_{c}^{0}\right)\right]+\int_{-r_{s}^{0}}^{r_{s}^{0}} d r^{\prime \prime} G\left(r, r^{\prime \prime}\right) \Omega_{s c}\left(r^{\prime \prime}\right),
$$

and

$$
\Omega_{c c}(r)=1+\frac{1}{\pi} \int_{-r_{s}^{0}}^{r_{s}^{0}} d r^{\prime \prime} \frac{\Omega_{s c}\left(r^{\prime \prime}\right)}{1+\left(r-r^{\prime \prime}\right)^{2}}
$$

From analysis of the form of the kernel function given in Eqs. (A.7), one straightforwardly finds that Eqs. (A.10)-(A.14) are equivalent to those that define the entries of the conformal-field theory dressed charge matrix of Ref. [25] and the transposition of that of Eq. [28]. Thus, this shows that the four parameters $\xi_{\alpha \alpha^{\prime}}^{1}$ given in (A.9) are indeed the entries of these matrices.

Finally, if one uses similar procedures for the four parameters $\xi_{\alpha \alpha^{\prime}}^{0}$, one finds that they are the entries of the transposition of the inverse of the conformal-field theory dressed charge matrix of Ref. [25] and the inverse of that of Eq. [28].

The quantity $2 \Delta_{\alpha}^{\iota}$ given in Eq. (25) has the same expression as the conformal dimension of the $\alpha, \iota$ primary field, provided that the four parameters $\xi_{\alpha \alpha^{\prime}}^{1}$ (and four parameters $\xi_{\alpha \alpha^{\prime}}^{0}$ ) are the entries of the conformal-field theory dressed charge matrix (and the entries of the transposition of the inverse of the conformal-field theory dressed charge matrix). Thus, we conclude that such a quantity, which was obtained by considering the low-energy limit of the general functional given in Eq. (24), is indeed the conformal dimension of the $\alpha, \iota$ primary field of the 1D Hubbard model $\alpha$ conformal-field theory.

\section{References}

[1] J. Voit, Rep. Prog. Phys. 58 (1995) 977.

[2] S. Tomonaga, Prog. Theor. Phys. 5 (1950) 544.

[3] J.M. Luttinger, J. Math. Phys. 14 (1963) 1154.

[4] I. Hiroyoshi, et al., Nature 426 (2003) 540.

[5] M. Bockrath, Nature 426 (2003) 511.

[6] O.M. Auslaender, et al., Science 308 (2005) 88.

[7] T. Lorenz, M. Hofmann, M. Grun̈inger, A. Freimuth, G.S. Uhrig, M. Dumm, M. Dressel, Nature 418 (2002) 614.

[8] P. Segovia, D. Purdie, M. Hengsberger, Y. Baer, Nature 402 (1999) 504.

[9] A. Recati, P.O. Fedichev, W. Zwerger, P. Zoller, Phys. Rev. Lett. 90 (2003) 020401.

[10] H. Basista, et al., Phys. Rev. B 42 (1990) 4088.

[11] R. Claessen, M. Sing, U. Schwingenschlögl, P. Blaha, M. Dressel, C.S. Jacobsen, Phys. Rev. Lett. 88 (2002) 096402; M. Sing, U. Schwingenschlögl, R. Claessen, P. Blaha, J.M.P. Carmelo, L.M. Martelo, P.D. Sacramento, M. Dressel, C.S. Jacobsen, Phys. Rev. B 68 (2003) 125111.

[12] E.H. Lieb, F.Y. Wu, Phys. Rev. Lett. 20 (1968) 1445.

[13] M. Takahashi, Prog. Theor. Phys. 47 (1972) 69; P.B. Ramos, M.J. Martins, J. Phys. A 30 (1997) L195.

[14] J.M.P. Carmelo, K. Penc, D. Bozi, Nucl. Phys. B 725 (2005) 421; J.M.P. Carmelo, K. Penc, D. Bozi, Nucl. Phys. B 737 (2006) 351, Erratum.

[15] J.M.P. Carmelo, K. Penc, cond-mat/0311075.

[16] J.M.P. Carmelo, K. Penc, L.M. Martelo, P.D. Sacramento, J.M.B. Lopes dos Santos, R. Claessen, M. Sing, U. Schwingenschlögl, Europhys. Lett. 67 (2004) 233; J.M.P. Carmelo, D. Bozi, P.D. Sacramento, K. Penc, in preparation.

[17] H. Benthien, F. Gebhard, E. Jeckelmann, Phys. Rev. Lett. 92 (2004) 256401.

[18] J.M.P. Carmelo, F. Guinea, K. Penc, P.D. Sacramento, Europhys. Lett. 68 (2004) 839. 
[19] K. Penc, K. Hallberg, F. Mila, H. Shiba, Phys. Rev. Lett. 77 (1996) 1390; K. Penc, K. Hallberg, F. Mila, H. Shiba, Phys. Rev. B 55 (1997) 15.

[20] J.M.P. Carmelo, J.M. Román, K. Penc, Nucl. Phys. B 683 (2004) 387.

[21] J.M.P. Carmelo, J. Phys.: Condens. Matter 17 (2005) 5517; J.M.P. Carmelo, D. Bozi, P.D. Sacramento, in preparation.

[22] H.J. Schulz, Phys. Rev. Lett. 64 (1990) 2831; J.M.P. Carmelo, A.H. Castro Neto, D.K. Campbell, Phys. Rev. Lett. 73 (1994) 926;

J.M.P. Carmelo, A.H. Castro Neto, D.K. Campbell, Phys. Rev. Lett. 74 (1995) 3089, Erratum.

[23] A.A. Belavin, A.M. Polyakov, A.B. Zamolodchikov, J. Stat. Phys. 34 (1984) 763; A.A. Belavin, A.M. Polyakov, A.B. Zamolodchikov, Nucl. Phys. B 241 (1984) 333.

[24] D. Boyanovsky, C.M. Naon, Riv. Nuovo Cimento 13 (1990) 2.

[25] F. Woynarovivh, J. Phys. A 22 (1989) 4243.

[26] M. Ogata, H. Shiba, Phys. Rev. B 41 (1990) 2326.

[27] N. Kawakami, S.K. Yang, Phys. Lett. A 148 (1990) 359.

[28] H. Frahm, V.E. Korepin, Phys. Rev. B 42 (1990) 10.

[29] M. Brech, J. Voit, H. Buttner, Europhys. Lett. 12 (1990) 289.

[30] H. Frahm, V.E. Korepin, Phys. Rev. B 43 (1991) 5653.

[31] M. Ogata, T. Sugiyama, H. Shiba, Phys. Rev. B 43 (1991) 8401.

[32] K. Penc, J. Sólyom, Phys. Rev. B 44 (1991) 12.

[33] J.M.P. Carmelo, P. Horsch, A.A. Ovchinnikov, Phys. Rev. B 45 (1992) 7899; J.M.P. Carmelo, P. Horsch, D.K. Campbell, A.H. Castro Neto, Phys. Rev. B 48 (1993) 4200.

[34] K. Penc, J. Sólyom, Phys. Rev. B 47 (1993) 6273.

[35] D. Jaksch, P. Zoller, Ann. Phys. 315 (2005) 52.

[36] Y. Chen, private communication.

[37] X.-J. Liu, P.D. Drummond, H. Hu, Phys. Rev. Lett. 94 (2005) 136406.

[38] J.M.P. Carmelo, K. Penc, cond-mat/0508704.

[39] O.J. Heilmann, E.H. Lieb, Ann. N.Y. Acad. Sci. 172 (1971) 583.

[40] C.N. Yang, Phys. Rev. Lett. 63 (1989) 2144.

[41] Y. Mizuno, K. Tsutsui, T. Tohyama, S. Maekawa, Phys. Rev. B 62 (2000) R4769.

[42] J.M.P. Carmelo, P.D. Sacramento, Phys. Rev. B 68 (2003) 085104.

[43] J.M.P. Carmelo, A.H. Castro Neto, Phys. Rev. Lett. 70 (1993) 1904; J.M.P. Carmelo, A.H. Castro Neto, D.K. Campbell, Phys. Rev. B 50 (1994) 3667; J.M.P. Carmelo, A.H. Castro Neto, D.K. Campbell, Phys. Rev. B 50 (1994) 3683.

[44] J.M.P. Carmelo, K. Penc, cond-mat/0508209.

[45] V. Meden, K. Schönhammer, Phys. Rev. B 46 (1992) 15.

[46] J. Voit, Phys. Rev. B 47 (1993) 6740;

J. Voit, J. Phys.: Condens. Matter 5 (1993) 8305.

[47] K. Schönhammer, V. Meden, Phys. Rev. B 47 (1993) 16.

[48] J.M.P. Carmelo, N.M.R. Peres, Phys. Rev. B 56 (1997) 3717.

[49] D. Baeriswyl, J. Carmelo, K. Maki, Synth. Met. 21 (1987) 271; N. Peres, J.M.P. Carmelo, D.K. Campbell, A.W. Sandvik, Z. Phys. B 103 (1997) 217.

[50] H. Bethe, Z. Phys. 71 (1931) 205; E.H. Lieb, W. Liniger, Phys. Rev. 130 (1963) 1605; P.-A. Bares, J.M.P. Carmelo, J. Ferrer, P. Horsch, Phys. Rev. B 46 (1992) 14624. 